\title{
BMJ Open Factors influencing the decision to convey or not to convey elderly people to the emergency department after emergency ambulance attendance: a systematic mixed studies review
}

\author{
Johan Oosterwold, ${ }^{1,2}$ Dennis Sagel, ${ }^{3}$ Sivera Berben, ${ }^{4,5,6}$ Petrie Roodbol, ${ }^{1}$ \\ Manda Broekhuis ${ }^{7}$
}

To cite: Oosterwold J, Sagel D, Berben S, et al. Factors influencing the decision to convey or not to convey elderly people to the emergency department after emergency ambulance attendance: a systematic mixed studies review. BMJ Open 2018;8:e21732. doi:10.1136/ bmjopen-2018-021732

- Prepublication history and additional material for this paper are available online. To view these files, please visit the journal online (http://dx.doi org/10.1136/bmjopen-2018021732).

Received 16 January 2018 Revised 16 July 2018 Accepted 18 July 2018
Check for updates

(C) Author(s) (or their employer(s)) 2018. Re-use permitted under CC BY-NC. No commercial re-use. See rights and permissions. Published by BMJ.

For numbered affiliations see end of article.

Correspondence to Mr. Johan Oosterwold; j.oosterwold@umcg.nl

\section{ABSTRACT}

Background The decision over whether to convey after emergency ambulance attendance plays a vital role in preventing avoidable admissions to a hospital's emergency department (ED). This is especially important with the elderly, for whom the likelihood and frequency of adverse events are greatest.

Objective To provide a structured overview of factors influencing the conveyance decision of elderly people to the ED after emergency ambulance attendance, and the outcomes of these decisions.

Data sources A mixed studies review of empirical studies was performed based on systematic searches, without date restrictions, in PubMed, CINAHL and Embase (April 2018). Twenty-nine studies were included.

Study eligibility criteria Only studies with evidence gathered after an emergency medical service (EMS) response in a prehospital setting that focused on factors that influence the decision whether to convey an elderly patient were included.

Setting Prehospital, EMS setting; participants to include EMS staff and/or elderly patients after emergency ambulance attendance.

Study appraisal and synthesis methods The Mixed Methods Appraisal Tool was used in appraising the included articles. Data were assessed using a 'best fit' framework synthesis approach.

Results ED referral by EMS staff is determined by many factors, and not only the acuteness of the medical emergency. Factors that increase the likelihood of nonconveyance are: non-conveyance guidelines, use of feedback loop, the experience, confidence, educational background and composition (male-female) of the EMS staff attending and consulting a physician, EMS colleague or other healthcare provider. Factors that boost the likelihood of conveyance are: being held liable, a lack of organisational support, of confidence and/or of baseline health information, and situational circumstances. Findings are presented in an overarching framework that includes the impact of these factors on the decision's outcomes. Conclusion Many non-medical factors influence the ED conveyance decision after emergency ambulance attendance, and this makes it a complex issue to manage.
Strengths and limitations of this study

- The broad and empirical nature of the study has made it possible to identify multiple factors that influence the referral decision by emergency medical service staff after ambulance emergency attendance, and the outcomes of this decision.

- Building on existing general decision-making frameworks, an overarching framework was developed that proved helpful in structuring the influential factors identified.

- A weakness is that not all of the factors identified can be definitely related to the elderly population because, in many studies, the elderly formed part of a broader study population, and the results were not specified by age group.

- The low methodological quality in some of the studies and the considerable age of some of them are limitations of the study.

\section{INTRODUCTION}

Rationale

An increasing demand for emergency medical service (EMS) responses is noticeable in many developed countries. ${ }^{1-4}$ The demand is highest with people aged over 65, and exponentially grows with increasing age. ${ }^{4-7}$ These elderly people need to get appropriate care after ambulance attendance, and this may not always be referral to a hospital's emergency department (ED). If EMS staff decide that ED attendance is not necessary, the patient can be left at home or referred to another healthcare facility. The possibilities vary by country, and their use is influenced by protocols, protocol adherence and alternative pathways. ${ }^{8-12}$ Both the increase in numbers of older people and the demand for EMS set challenges for future patient safety and providing the best possible healthcare. $^{13}$ 
Non-conveyance after an emergency ambulance response is an increasing trend in many West-European countries. ${ }^{14}{ }^{15}$ Non-conveyance can partly be attributed to patient refusals, minor injuries that are easy to handle and the death of patients. Incorrect decisions by EMS staff on not to convey patients to the ED can lead to health-threatening situations and even to death. ${ }^{16-19}$ Referral to the ED may result in overcrowding and, especially for the elderly population, is associated with higher mortality, delays in receiving critical therapy, patient dissatisfaction, iatrogenic illness, functional decline and adverse events during care. ${ }^{20-25}$ Correct conveyance decision-making by ambulance staff is therefore relevant, but also very complex due to the many influencing factors. ${ }^{2627}$ Further, national protocols do not always provide adequate guidance to EMS staff in making conveyance decisions, and guidelines and protocols are not always followed. ${ }^{12} 192829$ Reasons for non-adherence to protocols are attributed to the individual professional, the organisation, external factors and protocol characteristics (Grol, cited in Ebben et al).$^{30}$ Due to the large variety in situations, EMS staff often have to rely on their own professional judgement. Factors such as the use of guidelines and protocols, patient preferences, experience of EMS staff, time aspects and the presence of carers can influence ambulance staff when deciding whether to take a patient to the ED. ${ }^{31}$

Whether EMS staff can adequately determine the medical necessity for an ED evaluation is not easy to define and to measure. A systematic review and meta-analysis showed that there is insufficient evidence to support paramedics determining the medical necessity for ambulance transport. ${ }^{32}$ A retrospective analysis of ED data showed that $7.1 \%$ of patients aged $75+$ taken there by ambulance were considered as non-urgent, with the largest number of non-urgent conveyances following falls. ${ }^{33}$ Currently, researchers are focusing on adequate, community-based, alternative referrals by EMS staff for older people who have fallen. ${ }^{34} 35$

National protocols can guide EMS staff in making a decision over the conveyance or non-conveyance of an elderly person after an emergency ambulance call, but these protocols cannot cover the full scope of practice. Other factors also influence the conveyance decision-making process in which negotiation or joint decision-making between EMS staff, the patient and sometimes their family in deciding what is best for the patient can also play a pivotal role. ${ }^{193637}$ In the future, the growing ageing population will have major consequences for the utilisation of EMS and so the conveyance decision, to the ED or elsewhere, after emergency ambulance attendance is of growing importance. Insight into factors that influence this conveyance decision-making is especially important for the population of elderly because avoidable admissions may result in functional decline, iatrogenic illness, adverse events, ED overcrowding, excessive interventions and high healthcare costs. ${ }^{38}$ To increase knowledge about factors that may influence the conveyance decision for the specific group of elderly vulnerable people, after EMS attendance, there is a need for a full overview of these factors and the impact of the decision.

\section{Objectives}

The aim of this study is to provide an overview of those factors that influence the decision whether or not to convey an elderly person to the ED after ambulance attendance and the outcomes of such decisions. The findings will be summarised in a conceptual framework and are intended to inform practice, policy-makers and future researchers. They can also serve as a basis for developing future EMS conveyance decision-making guidelines for vulnerable elderly people, where special attention is paid to minimising the risk of inappropriate conveyance and use of EMS and ED resources, adverse outcomes and medical legal consequences.

\section{METHOD}

A systematic mixed-studies review (MSR) was chosen to synthesise primary qualitative, quantitative and mixedmethods research studies. ${ }^{39}$ The integrated design selected is appropriate for complex and context-sensitive interventions, and can provide a deep and highly practical understanding of phenomena in the health sciences. ${ }^{40}$ This MSR follows recognised guidelines for systematic mixed-studies reviews. ${ }^{39}$

\section{Eligibility criteria}

Studies were included if they contained empirical evidence on one or more factors that influenced the conveyance or non-conveyance decision to an ED for an elderly person after being attended by ambulance personnel. In more detail, studies were incorporated if they specifically addressed elderly patients, elderly people were part of a broader age group (eg, all adults), the factors considered could be linked to elderly patients (eg, end-of-life situations, falls) or when general factors were identified that affected all age groups (eg, EMS staff-related factors). Searches were not restricted by publication date or by country, although only publications written in English, Dutch or German were eligible for inclusion. Detailed inclusion and exclusion criteria are provided in online supplementary appendix 1 .

\section{Information sources}

Three database searches (PubMed, Embase and CINAHL) were executed in October 2016, and these were updated in April 2018 to identify any relevant research published since the initial search. The search terms covered three areas: (1) ambulance or emergency medical services, (2) 'conveyance or non-conveyance of patients' or 'treat and release' or 'referral and consultation', and (3) 'decision-making'. The research team performed a broad search in order to include all the potentially relevant articles, meaning that a high percentage of the initial list would not be relevant. Only peer-reviewed articles were included in order to ensure a generally accepted level 
of quality. The full electronic search strategy is shown in online supplementary appendix 2.

\section{Study selection}

In this systematic MSR, the support tool 'StArt'-State of the Art through systematic review-was used in the process of screening for relevant articles. ${ }^{41}$ All the articles retrieved $(n=2412)$ were checked by one researcher for duplicates and irrelevant studies, and these were removed; the latter phase employed the exclusion criteria shown in table 1. A second reviewer (MB) independently screened a small random sample (5\%), and there was full agreement on the accepted and rejected studies. Two reviewers (JO and DS) independently assessed the full texts of the remaining subset of 108 articles. Cohen's kappa was calculated to determine if there was agreement between the two reviewers. The strength of agreement was considered to be 'good', $\kappa=0.786$ (95\% CI 0.652 to 0.919 ), and differences were resolved by discussion. Finally, 29 articles were accepted for inclusion in the systematic literature review (figure 1).

\section{Data collection process}

One researcher (JO) extracted data from the included studies. Characteristics extracted included setting, aim of the study, study design and study population (table 1). Data were also extracted describing factors that influenced the conveyance decision after ambulance attendance. A brief summary of these factors and the subjective/objective outcomes of the decision are shown in table 2 .

\section{Appraisal}

One author (JO) assessed all the included articles and four authors (PR, DS, SB and MB) each assessed some of them using a multimethod appraisal tool (MMAT, version 2011). ${ }^{39} 42$ The MMAT has been tested for validity and been used in various systematic MSRs to evaluate the methodological quality by answering four questions regarding recruitment, randomisation (if applicable), appropriateness of outcome measures and attrition rate/ completeness of data. The final score reflects the number of criteria satisfied, varying from one criterion met (reported as $*$ ) to all criteria met $(* * * *)$. Any disagreements in ratings between reviewers were discussed until a consensus was reached.

\section{Synthesis of results}

In this systematic review, a 'best fit' framework was used as a starting point for data synthesis. ${ }^{43}$ Since no suitable framework existed for the topic studied, a 'best fit' framework was constructed based on two existing models, one describing the process of clinical decision-making by Gillespie and Peterson and the other, the Input-ProcessOutput (IPO) model of Steiner and Hackman. ${ }^{44-47}$

The Situated Clinical Decision-Making framework by Gillespie and Peterson is a tool that is often used to assist educators in analysing nursing students, or novice nurses, in their complex and multidimensional clinical decision-making process. ${ }^{44}$ It can also be applied within
EMS practice since these decisions are also made within a dynamic context, knowledge is used from multiple sources, is influenced by all that the profession brings to knowledge and experience and is supported by a range of thinking processes. ${ }^{44}$ The themes covered by the Situated Clinical Decision-Making framework were incorporated within an IPO model (figure 2).

Finally, the objective and subjective outcomes are added to the framework. The process of data extraction, coding and analysis in this MSR leads to a conceptual framework that describes the factors that actually influence the decision of conveyance, and the subjective and/or objective outcomes of such decisions.

\section{Patient and public involvement}

There was no involvement of patients and or public in this study.

\section{RESULTS}

\section{Study selection and characteristics}

This systematic literature review covers 29 articles all published between 1995 and 2018 with the majority $(\mathrm{n}=19)$ published after 2010. The studies were mostly carried out in the UK $(n=13)$ and the USA $(n=12)$. The four remaining studies were from Sweden, Poland, Australia and Iran. Sixteen of the studies used quantitative research designs, 12 were qualitative and only 1 study used mixed methods. There were eight studies which focused exclusively on elderly people (aged $\geq 65$ ), and in 10 studies, elderly people were part of a broader age group. In the remaining 11 studies, factors were identified that affected all age groups.

\section{Quality of the studies}

Using the quality criteria discussed earlier, four studies were classed as of low quality $(*$ or $* *),{ }^{48-51} 15$ as average $(* * *)^{52-66}$ and 10 as good $(* * * *) .{ }^{67-76}$ Nevertheless, we included all the studies in our analysis but ranked them according to their quality score within the conceptual framework. Ranking was done by taking the average of the MMAT score of the related articles per theme and categorising them as A ( $\geq 3$ asterisks), B ( $\geq 2$ and $<3$ asterisks) or $\mathrm{C}(<2$ asterisks $)$.

\section{Summarising and synthesis}

The analysis resulted in a table presenting a priori themes within the 'best fit' framework with the relevant specific factors and a short summary of these factors (table 1). If described in the reviewed papers, the subjective and/ or objective outcomes were also presented alongside the specific factors.

\section{Macro-level themes}

Governmental, societal and professional themes were identified in the literature that influenced the conveyance decision-making process. One study by Déziel concluded that private EMS services were more likely to convey a patient to the hospital than public EMS services 


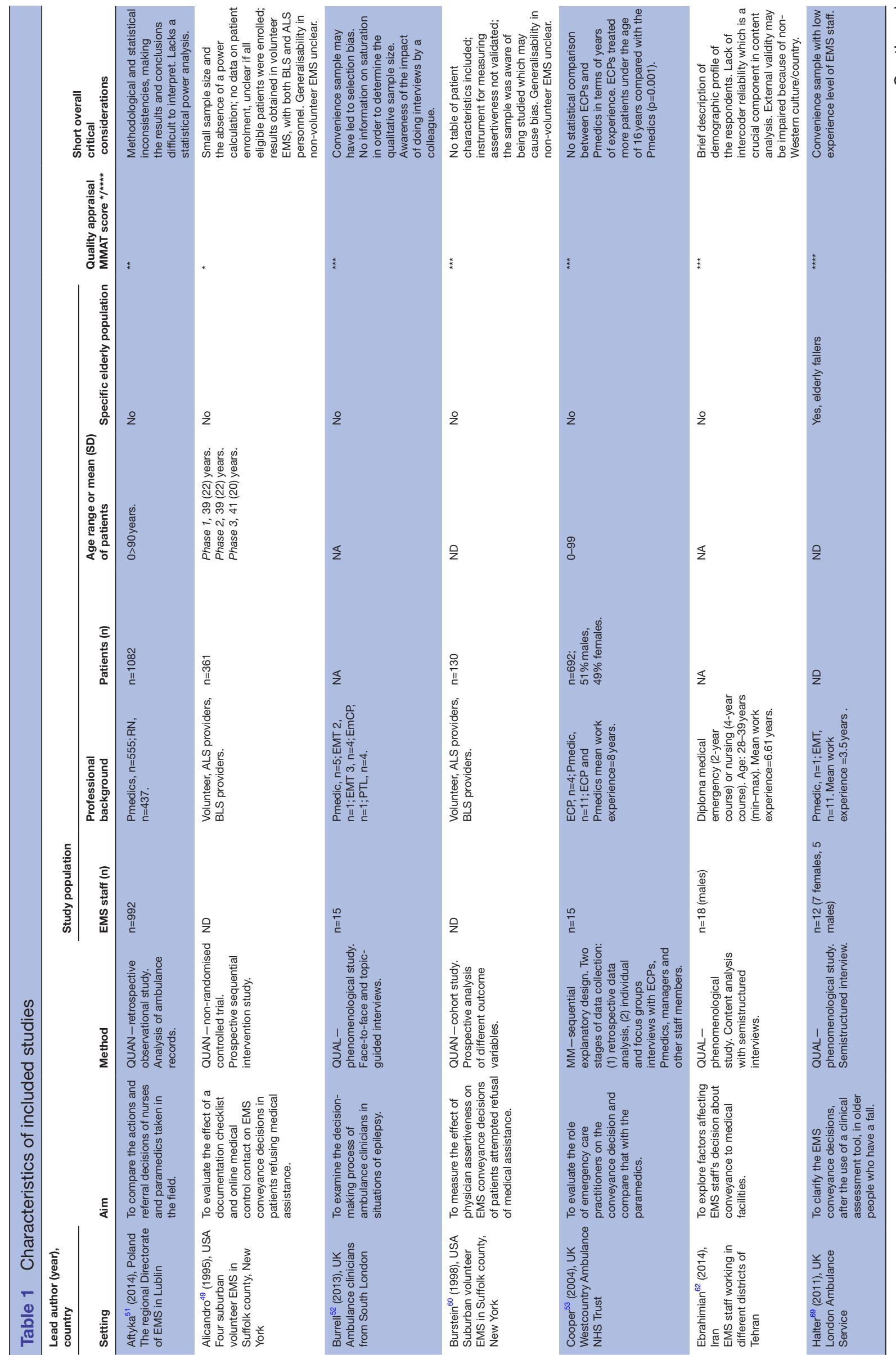




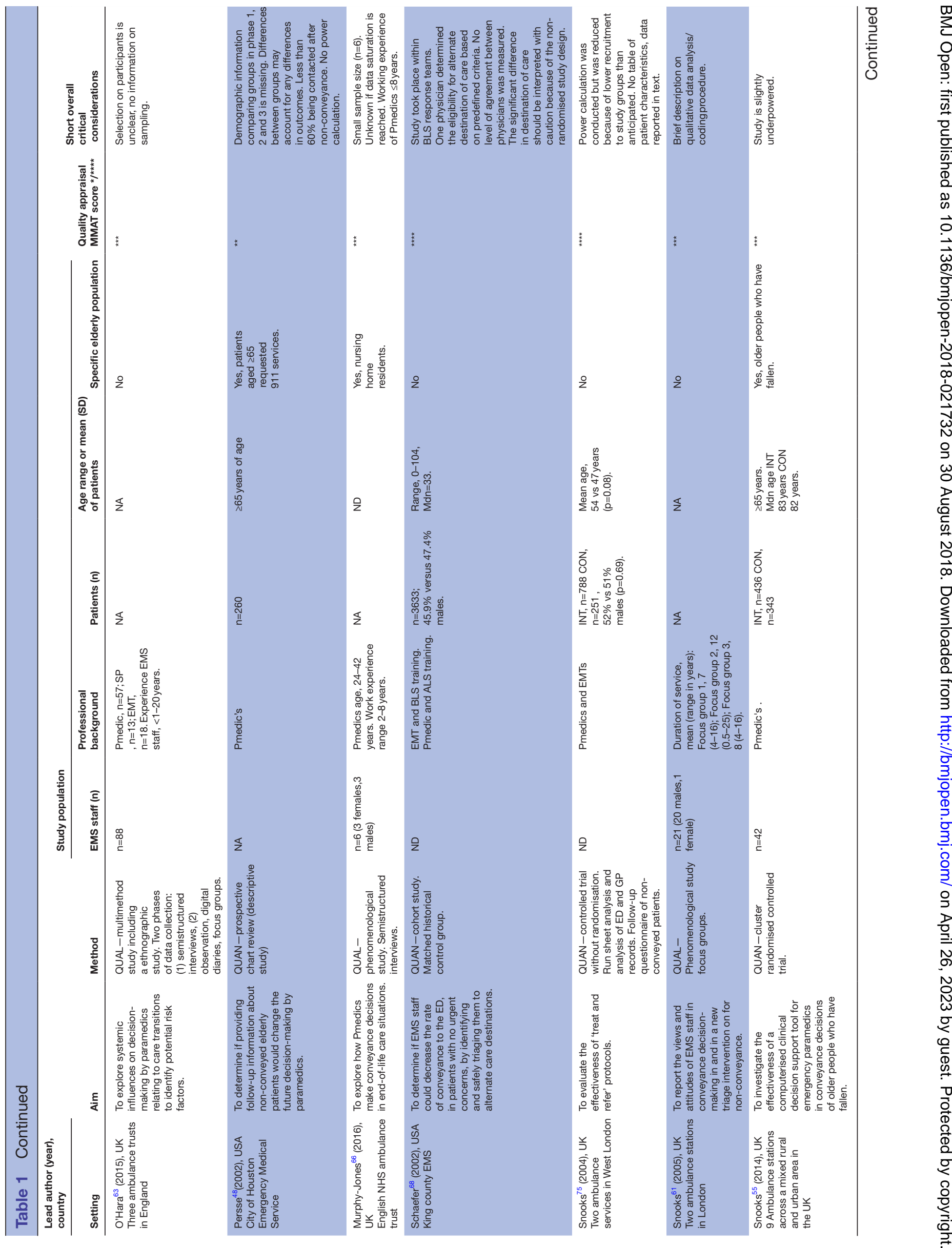




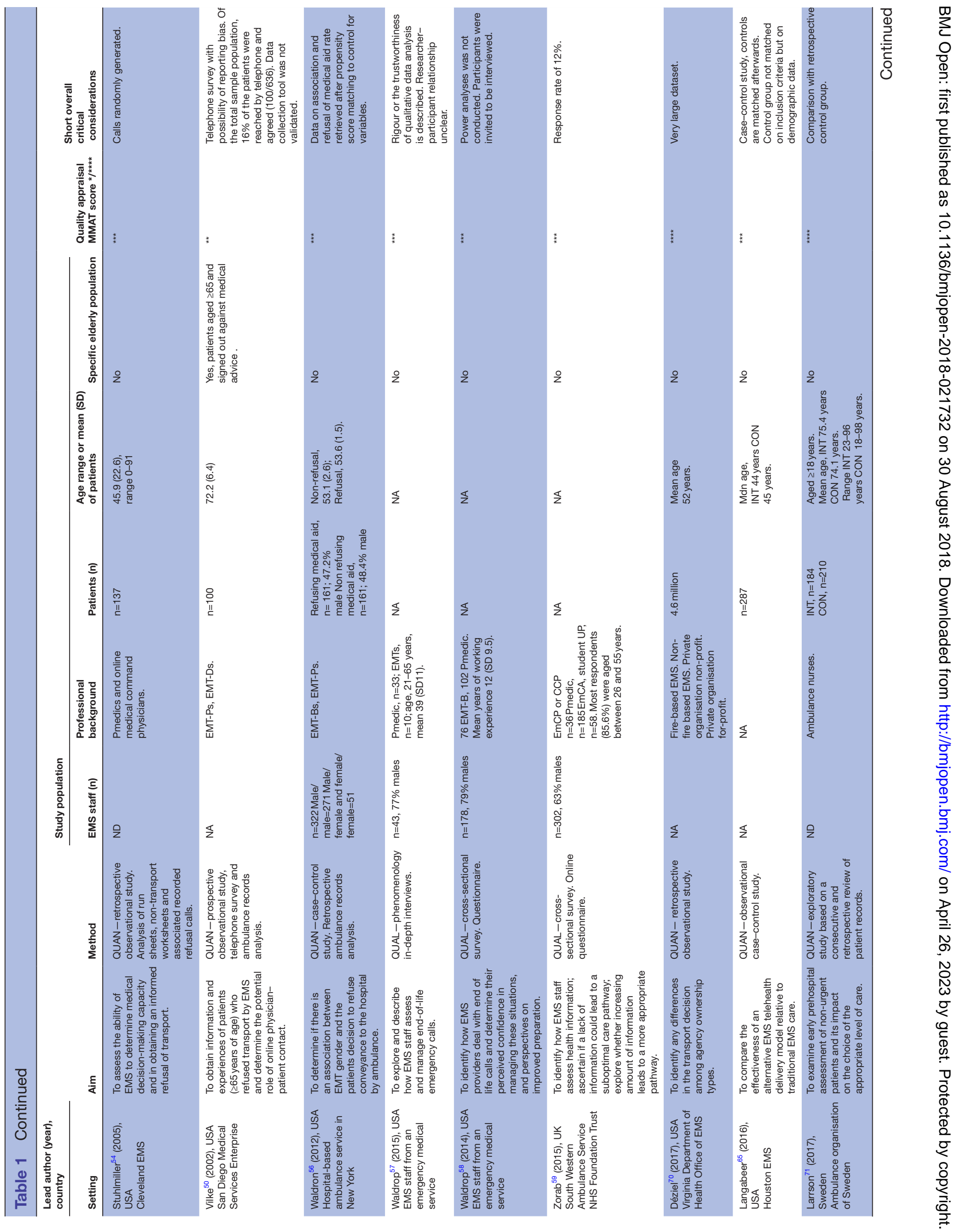




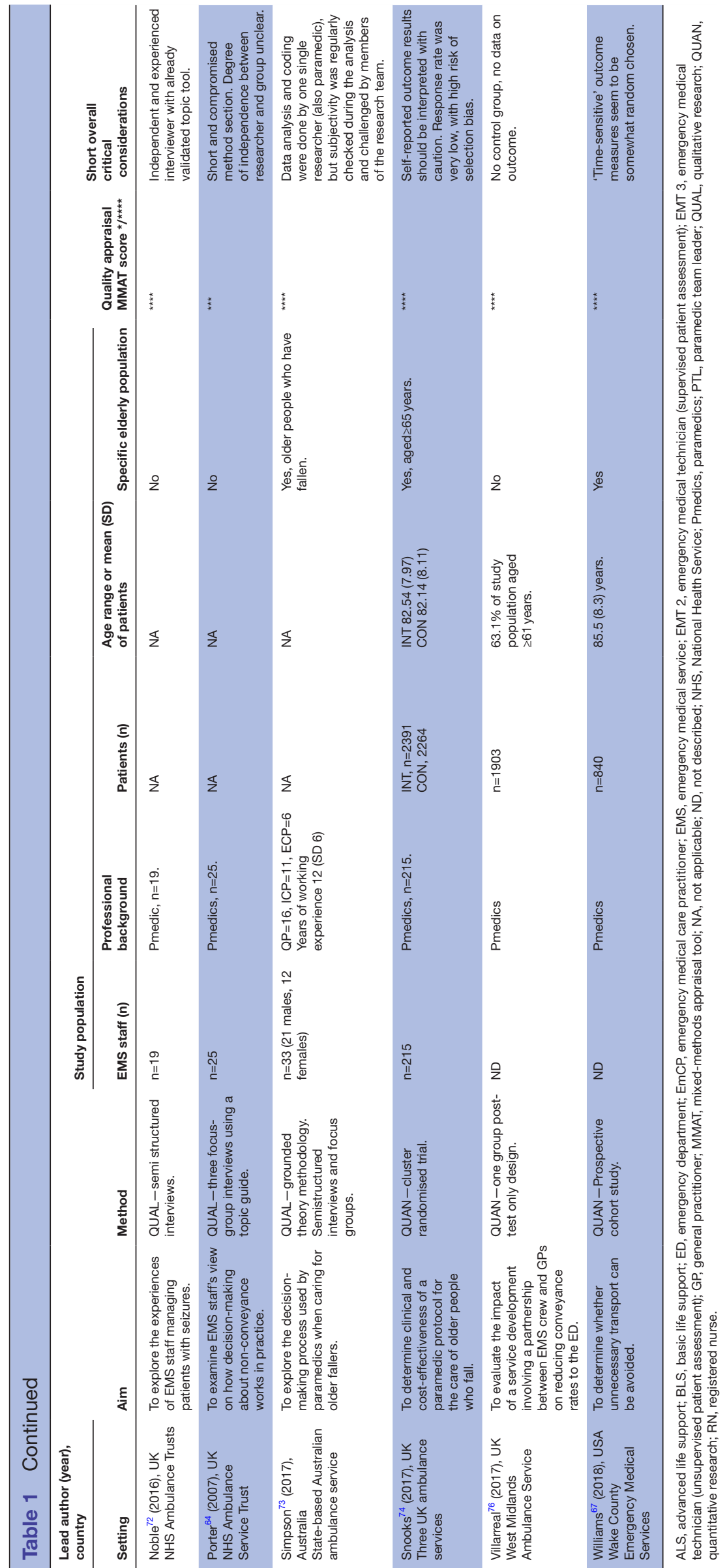

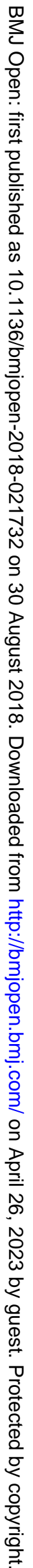




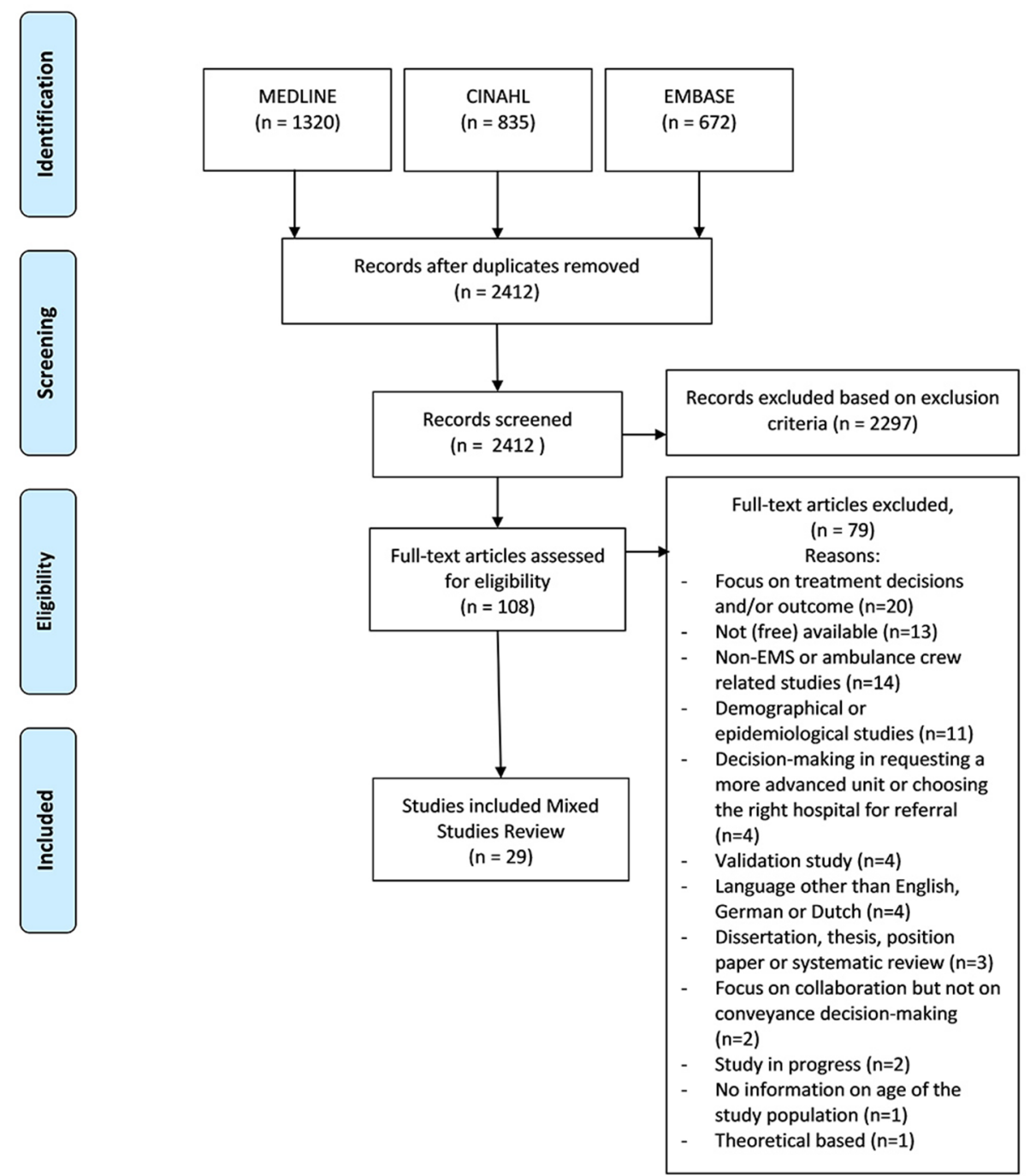

Figure 1 Preferred Reporting Items for Systematic Reviews and Meta-Analyses flow diagram of the selection process. EMS, emergency medical service.

(likelihood of conveyance by private EMS service is 4.5 times greater than with a public service).$^{70}$

Within the society theme, the factor 'Presence or absence of alternative care destinations for low-acuity diagnoses' was mentioned as an important reason for conveyance to the ED. ${ }^{52} 636872$ Where there were alternative destinations (other than referral to the hospital), Schaefer et al found a decrease in the proportion of non-acuity patients who were referred to the ED relative to a historical control group ( $51.8 \%$ vs $44.6 \%, \mathrm{p}=0.001$ ). No increase in medical morbidity resulted from this reduction in hospital referrals, and the patients with alternative care destinations were satisfied with their care $^{68}$

Within the profession theme, 'being held liable' was found to be an important factor leading to possibly unnecessary conveyance to the ED. ${ }^{526466}$ EMS staff feared being held responsible for a patient's welfare, and opted for the safe option of referral to the ED rather than 'treat and release'.

\section{Meso-level themes}

Three themes on the meso level had been identified as influencing the conveyance decision after an emergency ambulance call: 'EMS organisational structure', 'availability of appropriate resources and/or persons' and 'workload'. Most of the factors identified were within the 'EMS organisational structure' theme. Four studies $^{52} 636473$ reported that low confidence in the organisational support led to decisions reflecting minimising risk and thus conveyance to the ED. Operational demands, such as minimising on-scene time and reducing the number of conveyance rates, were factors in the decision-making process, but were counter-productive. Non-conveyance decisions are often more complex and time consuming and therefore increasing on-scene time. 61627273

An important factor within the 'availability of appropriate resources and/or persons' theme is the presence of clear directives or protocols. EMS staff indicated that conveyance protocols could give legitimacy to informal 


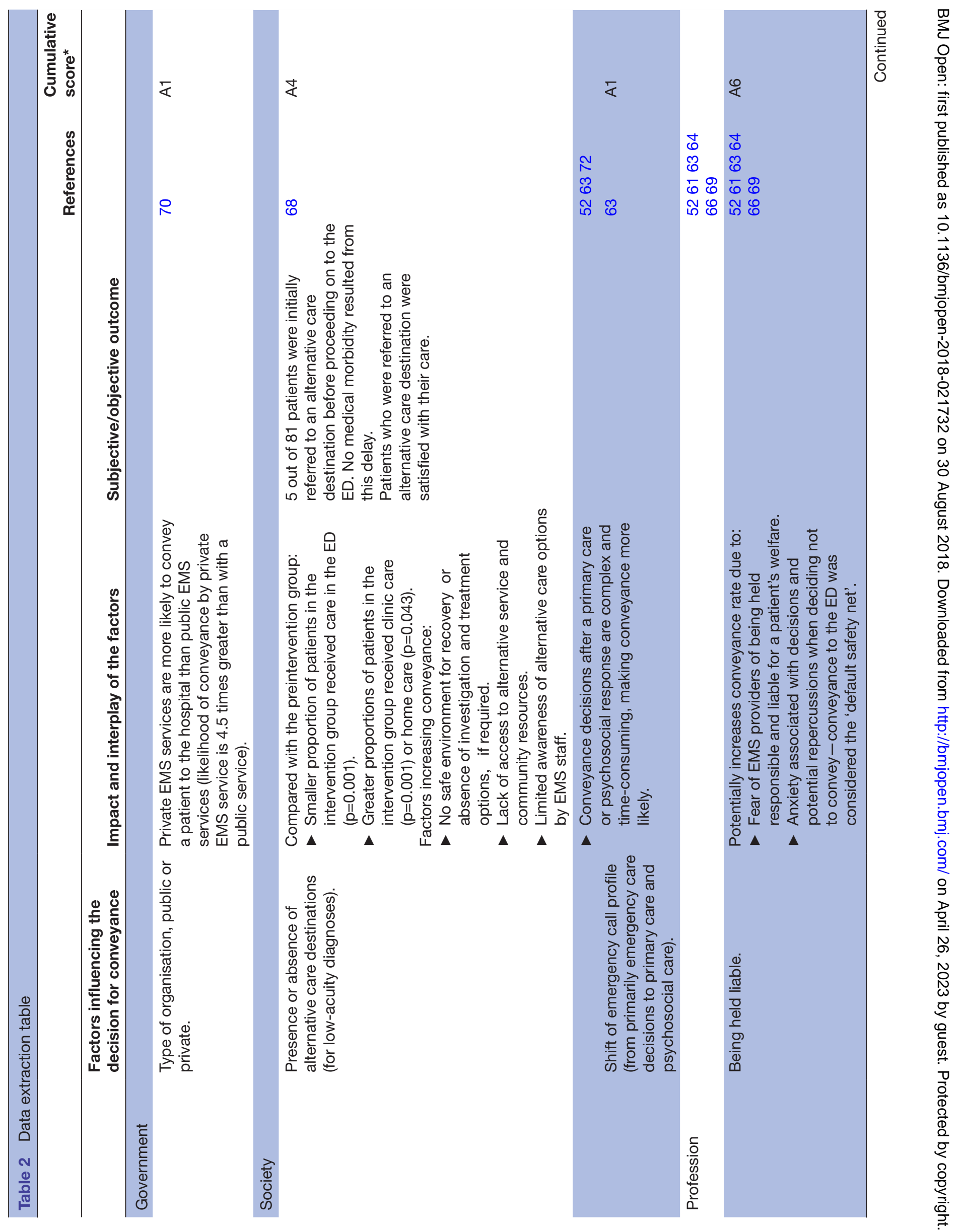




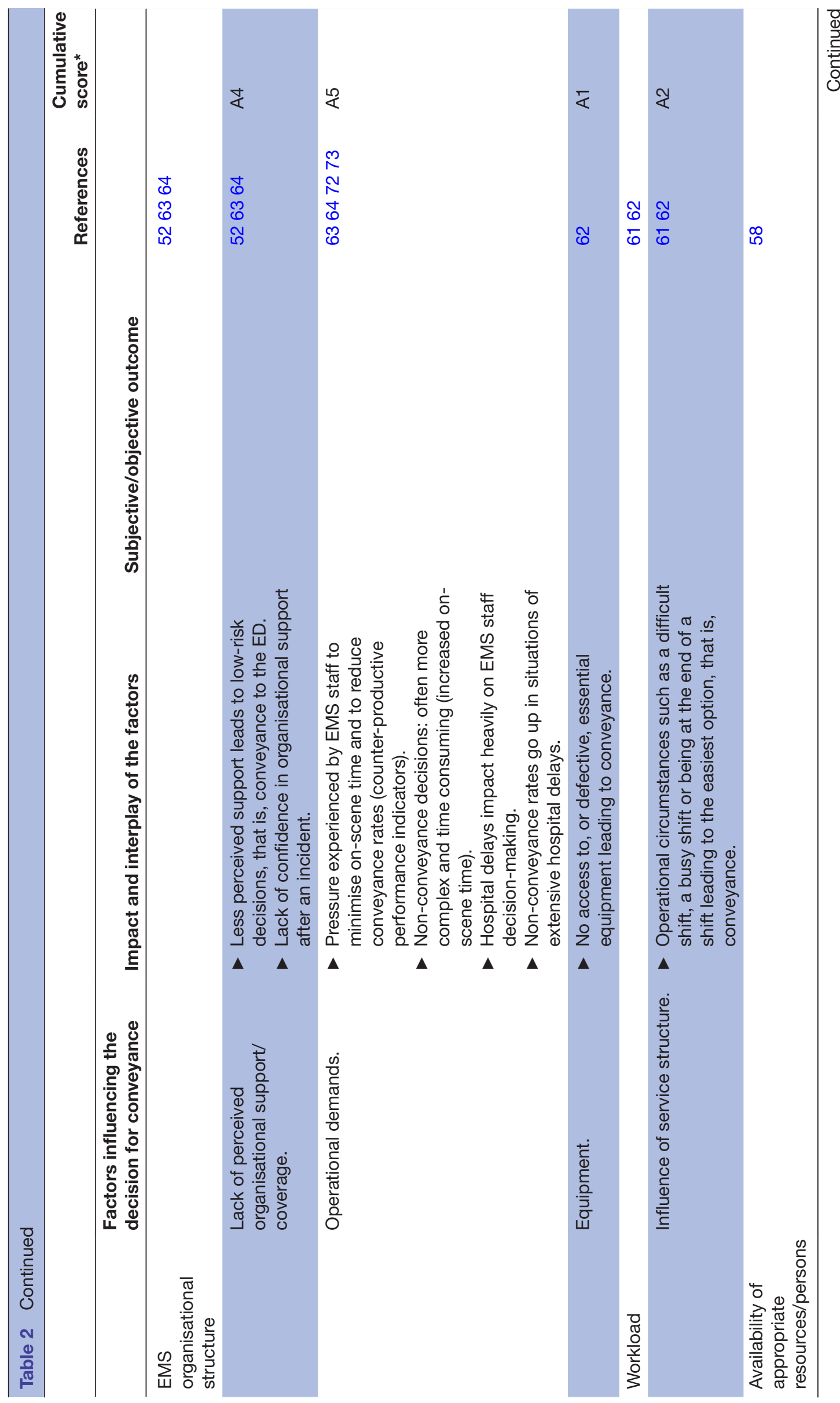

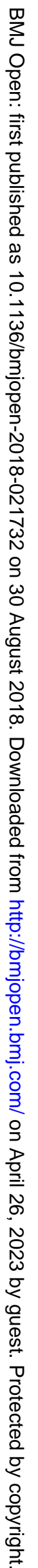




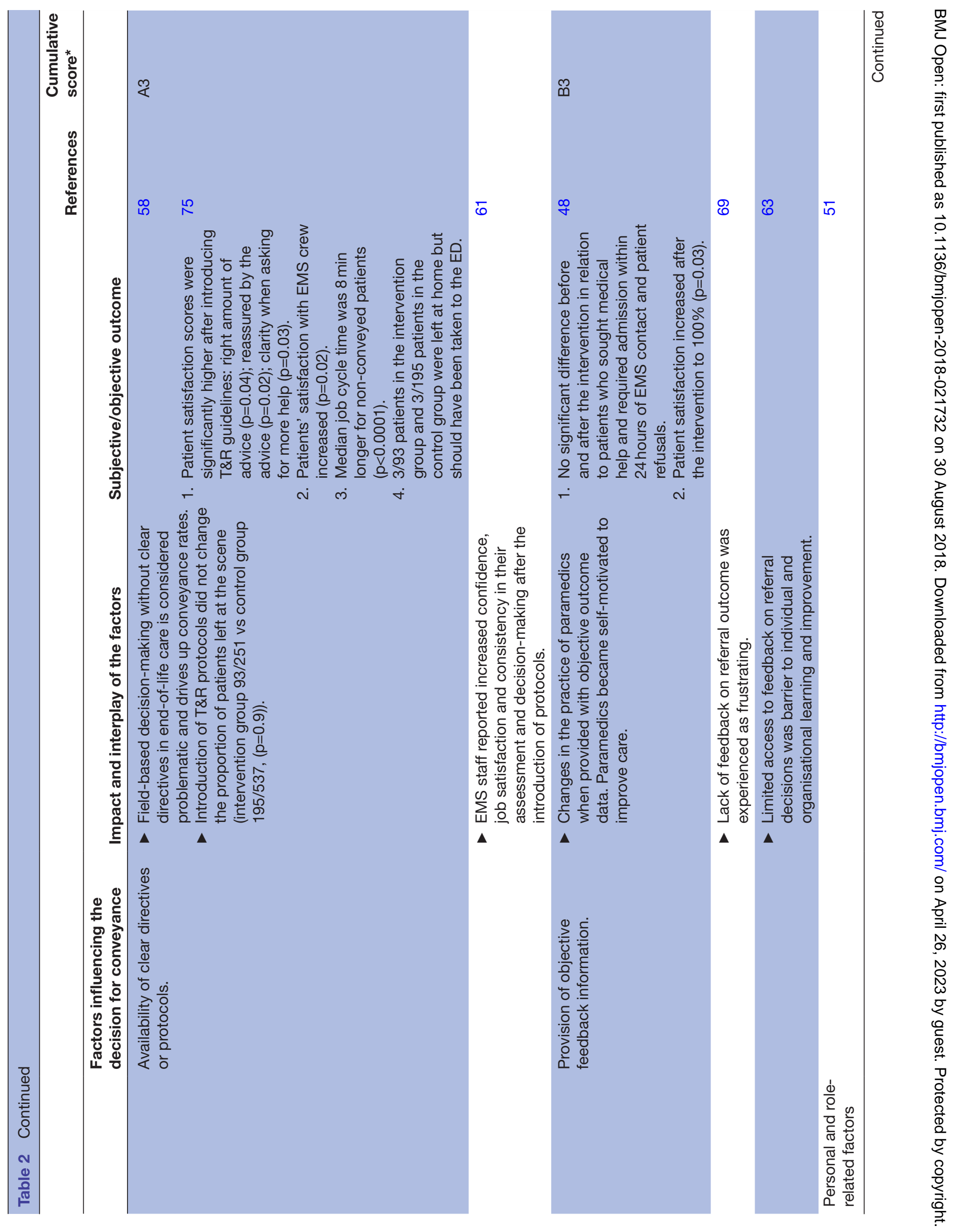




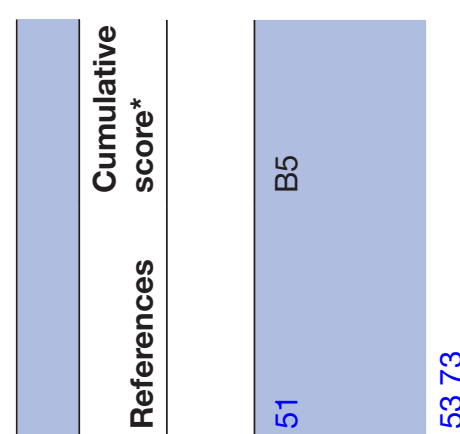

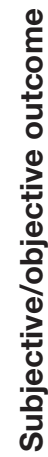

0 응

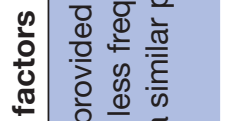

ब.

tᄂ

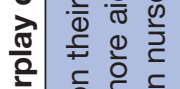

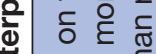

을 구

ত

竞

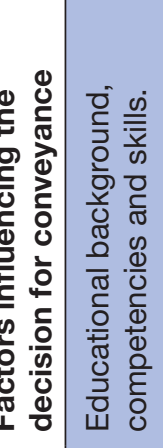

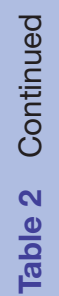

$\stackrel{1}{\infty}$

$\stackrel{\mathscr{D}}{\bar{D}}$

कृ

完

뜌웡

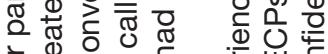

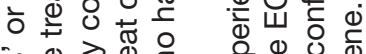

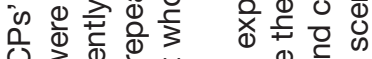

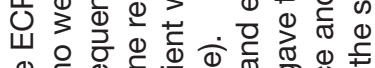

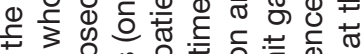

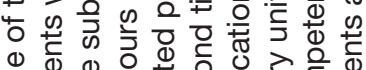

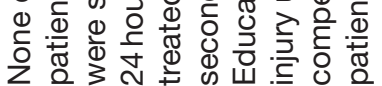

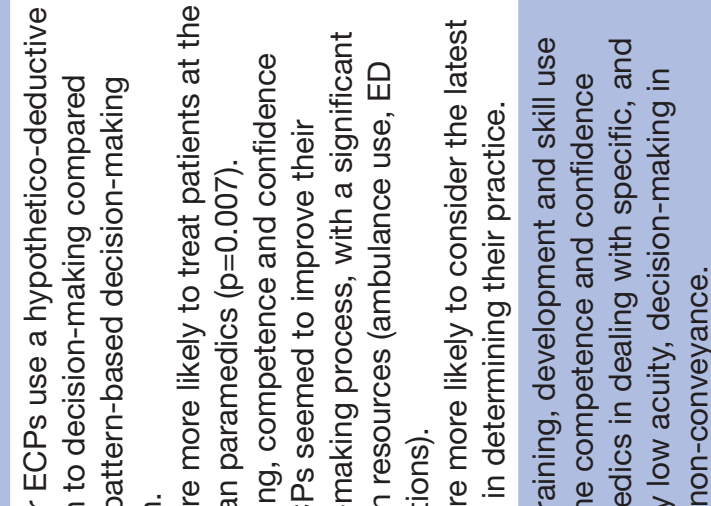

山유.

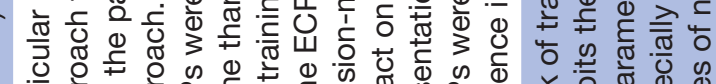

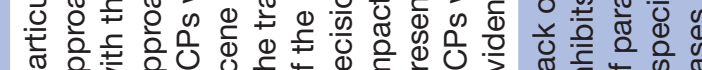

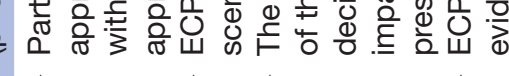
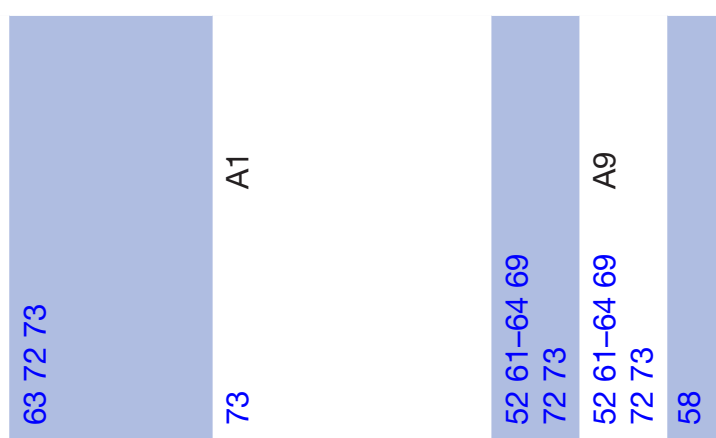

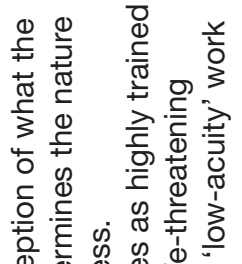
ه के 웡 으을 흔 这.

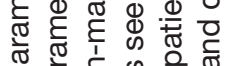

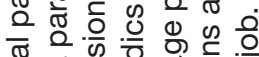

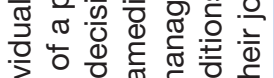

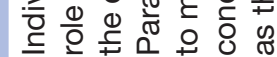

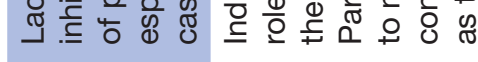

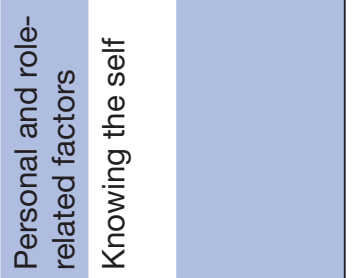




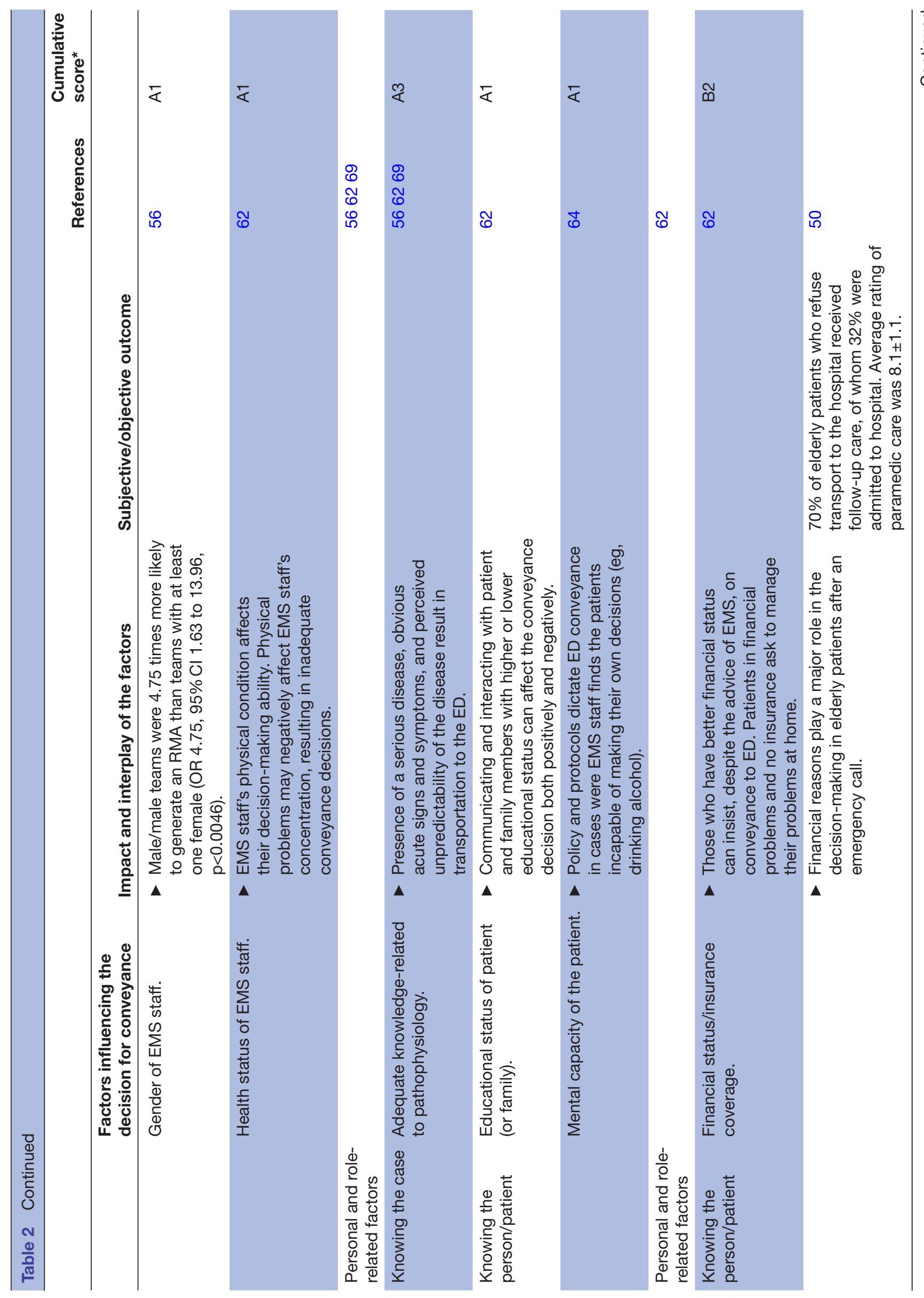




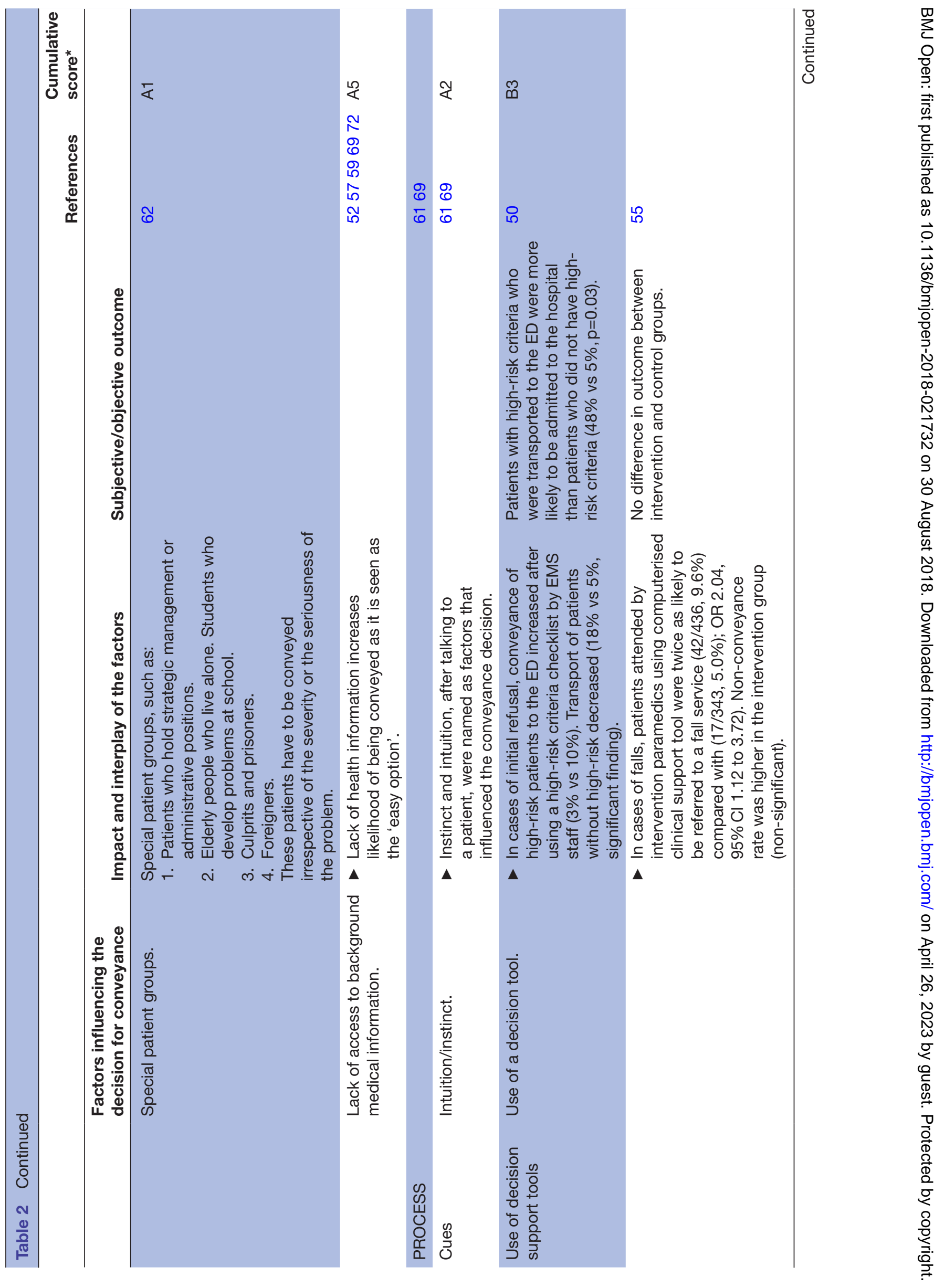




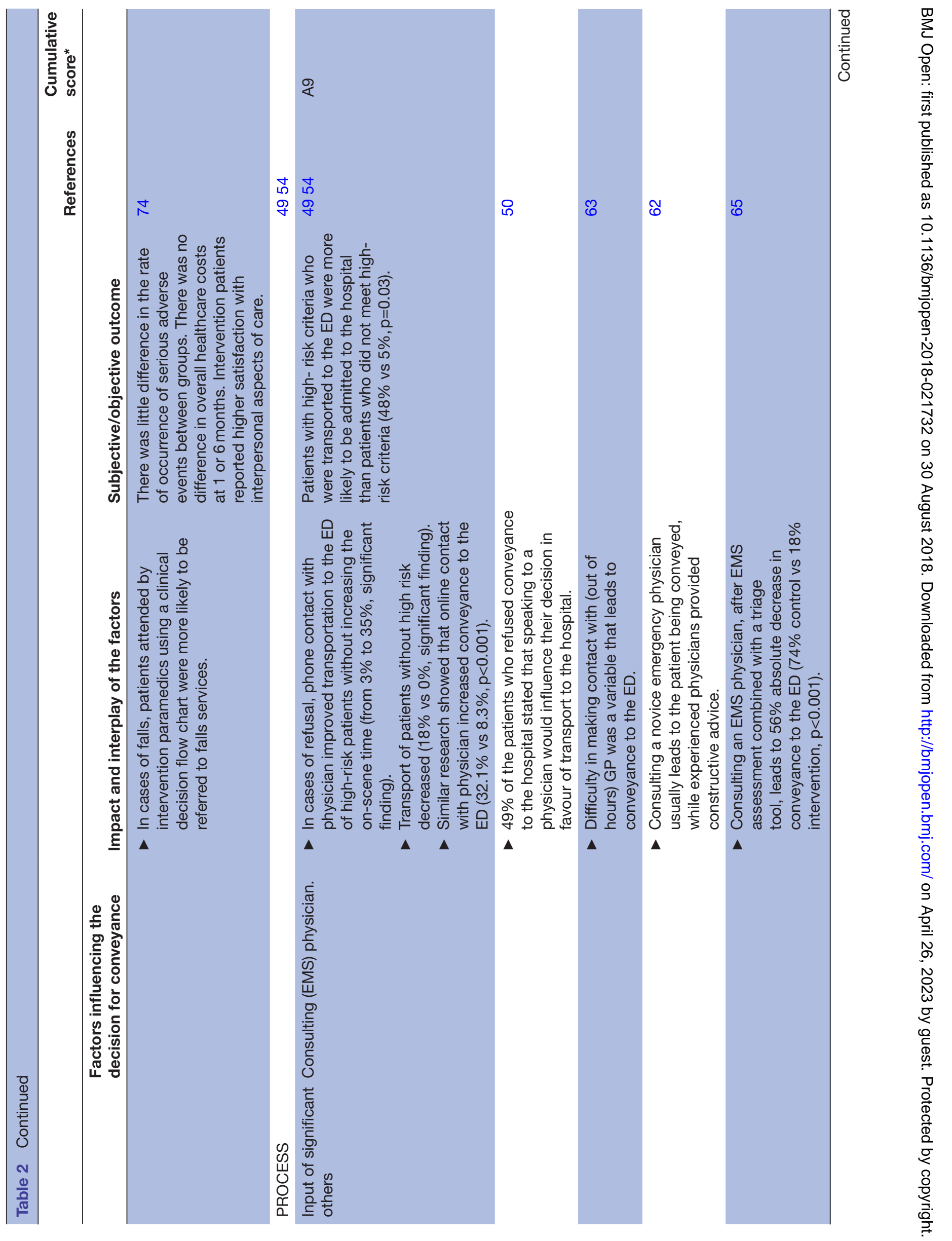




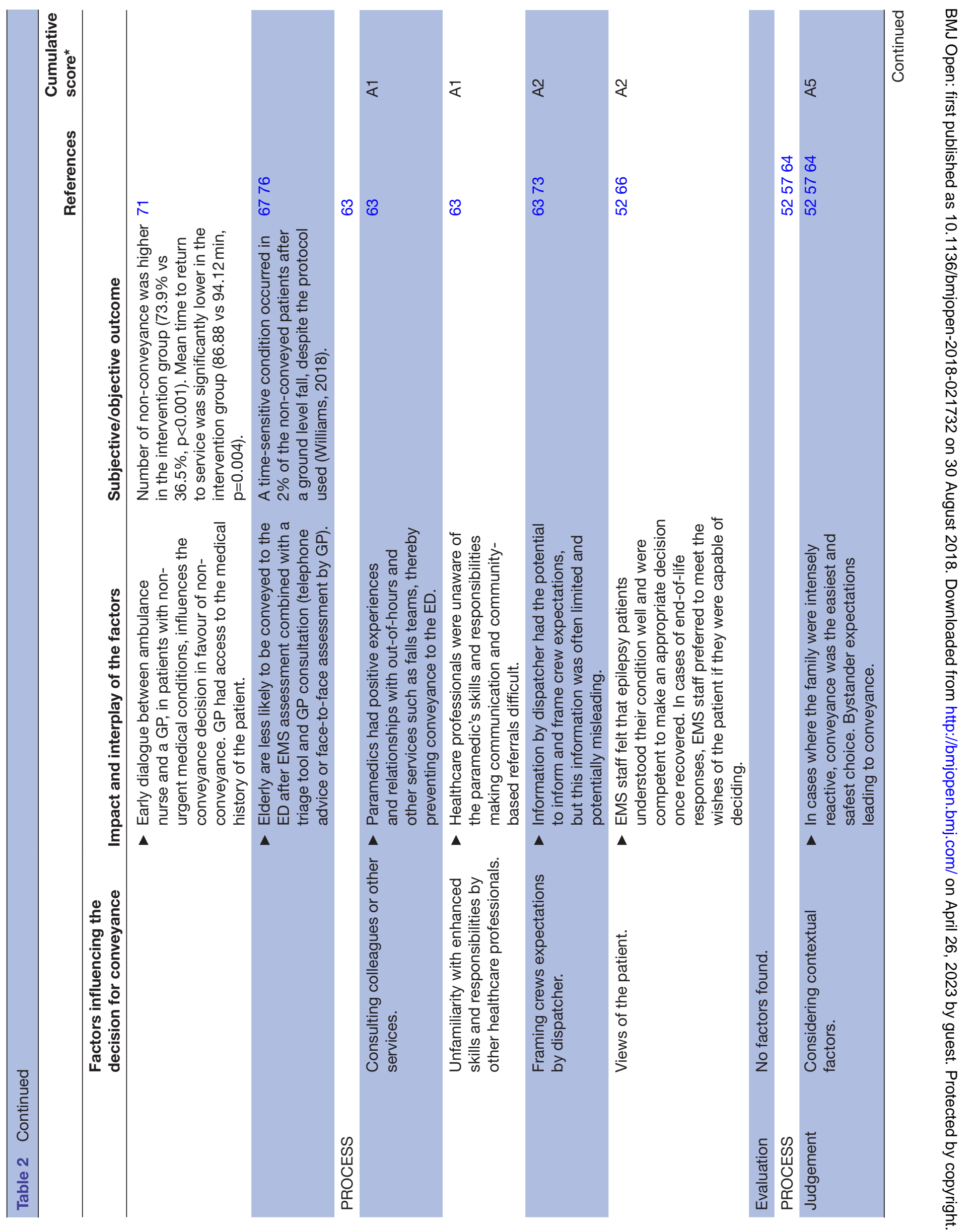




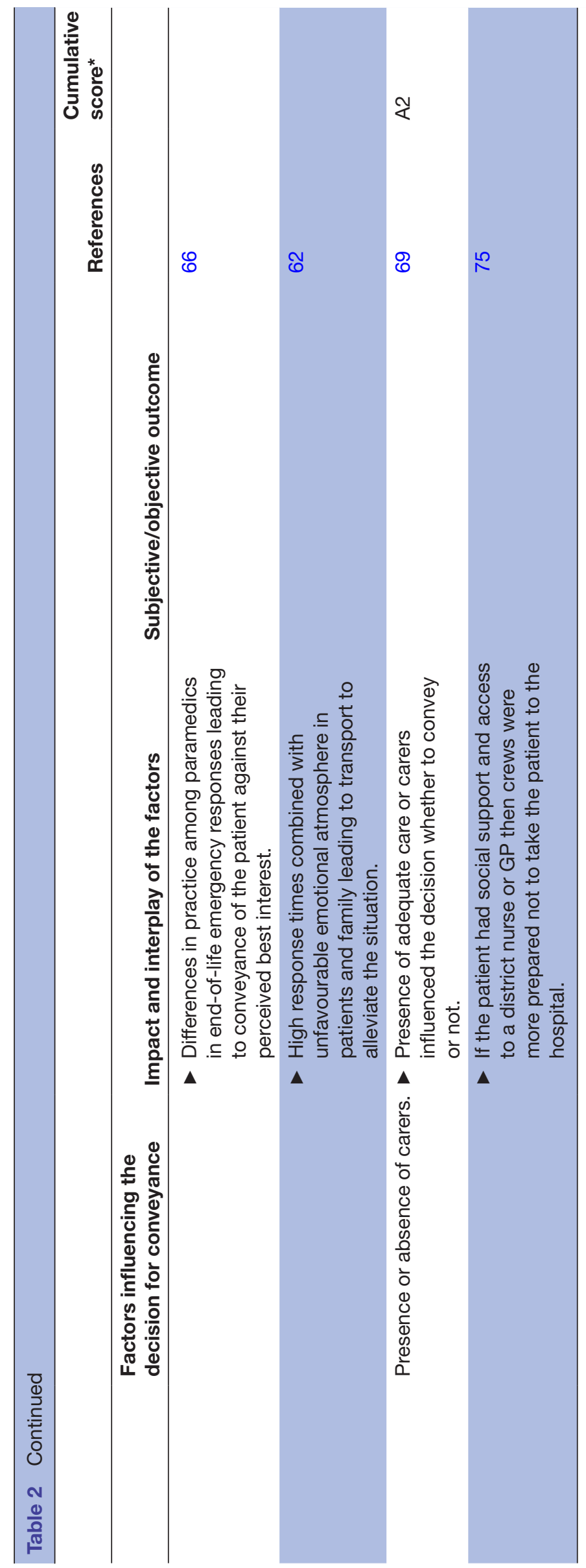

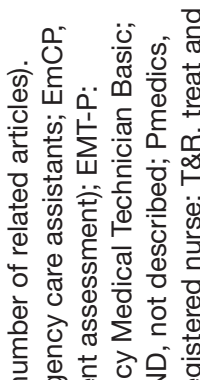

㠰

윰

$\underset{\vec{F}}{\stackrel{2}{+}}$

흠

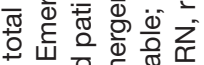

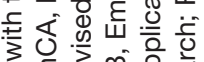

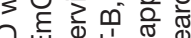

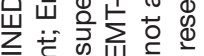

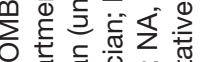

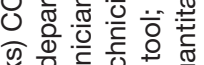

कै

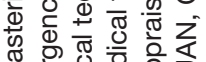

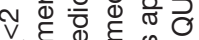

0 \&

के ये ठे

के

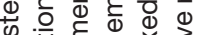

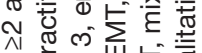

윤눙

离

ه

in

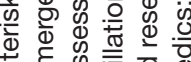

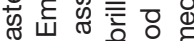

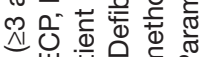

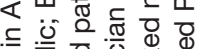

o

की

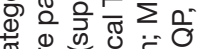

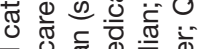

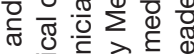

की

满 궁

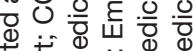

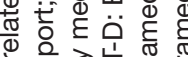

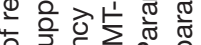

क क एँ

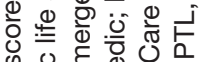

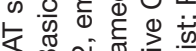

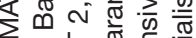

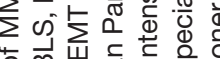

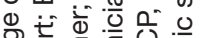

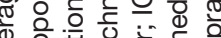

ส

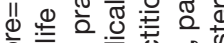

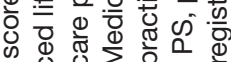

1)

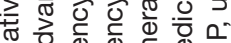

的要

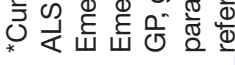




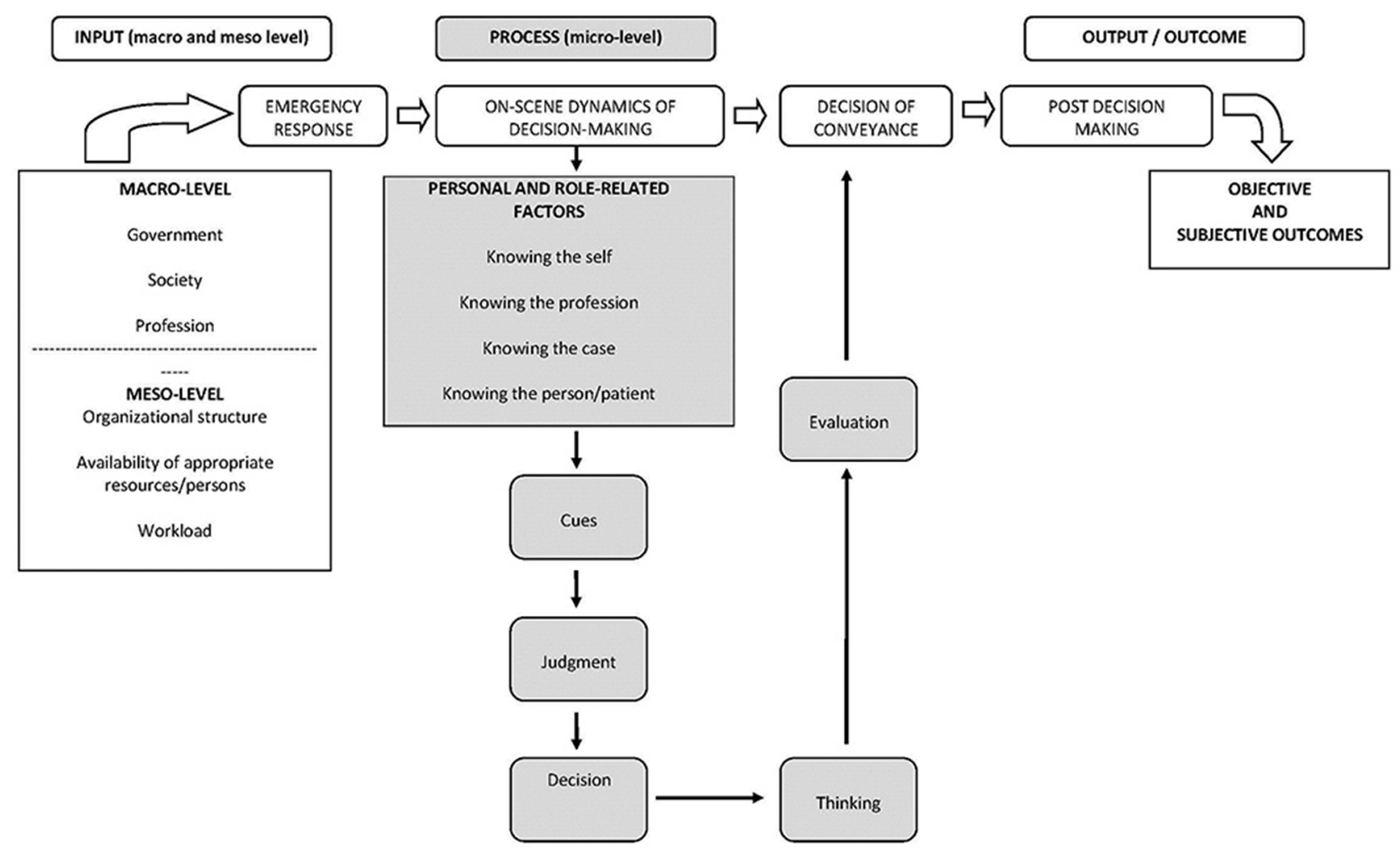

Figure 2 A priori theoretical framework of the decision-making process on conveyance by emergency medical service staff (based on Gillespie and Peterson, Steiner and Hackman). ${ }^{44} 464$

practice, but did not necessarily influence conveyance rates. ${ }^{61}$ Effects that were reported after the introduction of new guidelines/protocols were: higher patient satisfaction rates ${ }^{61}$ increased mean job-cycle time,${ }^{55}$ better documentation of clinical assessment, ${ }^{61}{ }^{75}$ and increased job satisfaction and confidence of EMS staff. ${ }^{61}$ Another factor found within this theme was making use of a 'feedback loop'. When EMS staff were provided with objective feedback information on non-conveyance responses, their self-motivation to improve care increased, ${ }^{48} 63$ and this led to individual and organisational learning. ${ }^{63}$ Under the workload theme, two studies found that attending incidents during difficult or busy shifts, or at the end of a shift, led to taking the easy option of conveying the patient to hospital. ${ }^{6162}$

\section{Micro-level themes: dynamics in the decision-making process}

The micro level consists of the knowledge that informs EMS staff on the scene, and can be subdivided into six themes: 'personal and role-related factors', 'cues', 'judgement', 'input of significant others', 'thinking' and 'evaluation'.

\section{Theme 1: personal and role-related factors}

In terms of personal and role-related factors, decision-making is informed by four knowledge-related aspects: 'knowing the self', 'knowing the profession', 'knowing the case' and 'knowing the person/patient'.

Most of the information uncovered from our review related to the 'knowing the self' aspect. Several factors influence the conveyance decision: their experience and confidence (where experience was reported as more important than training), ${ }^{58616264697273}$, previous negative experiences, ${ }^{52}{ }^{63}$ gender ${ }^{56}$ and the health status of the EMS staff. ${ }^{62}$ One study that examined the influence of EMS staff gender on non-conveyance due to patient refusal found that all-male teams were 4.75 times more likely to be confronted with a refusal of medical aid and subsequent conveyance to the ED than all-female and mixed-gender teams. ${ }^{56}$

Educational background, labelled as the 'knowing the profession', also influenced the conveyance decision. It has been reported that paramedics less frequently convey patients to a hospital than nurses. ${ }^{51}$ Cooper et al and Simpson et al reported that patients seen by an emergency care practitioner (ECP), someone who combines extensive nursing and paramedic skills, were less likely to be conveyed to the ED than those seen by paramedics. ${ }^{53} 73$ None of the articles investigating this topic provided information on objective outcomes linked to the educational background of the EMS providers. However, Cooper et al did note that there was no difference between paramedics and ECPs in terms of non-conveyed patients requiring subsequent conveyance to the ED within 24 hours. Simpson et al also reported extensively on paramedic role perception as a factor that influenced decision-making. Many felt that engagement in fall risk assessment or injury prevention did not fall within the scope of their function. ${ }^{73}$ 
Adequate pathophysiology knowledge was classified under the 'knowing the case' aspect. Here, recognition of the presence of a serious disease, obvious acute signs or perceived unpredictability of a disease resulted in direct conveyance to the ED. ${ }^{566269}$

Finally, five factors were linked to the 'knowing the person/patient' aspect. Patients with a better financial status were more likely to be conveyed to the ED. ${ }^{506}$ The majority of the elderly $(70 \%)$ who were denied conveyance to the ED because of their poor financial status did receive follow-up care, of which $32 \%$ were later admitted to a hospital. Furthermore, the 'educational status of the patient' and being a 'special case', such as elderly patients who lived alone, prisoners or foreigners, someone who had become incapable of making his/her own decisions were reported as influencing the conveyance decision. ${ }^{62}{ }^{64}$ Lastly, having access to the medical history and/or baseline health information influenced the conveyance decision. In the absence of such information, conveyance to the ED may be seen as the easiest and safest option. 5258596972

\section{Theme 2: cues}

Two studies described how intuition or 'instinct' influenced the conveyance decision. ${ }^{61} 69$ That is, a feeling based on previous work or clinical experience became a lesson that informed later decisions.

\section{Theme 3: use of decision support tools}

Use of a decision support tool increased the conveyance of patients to a specific service for those who had suffered falls rather than to the ED. ${ }^{61}{ }^{74}$ No differences in eventual outcomes between the two referral options were found. The EMS staff indicated that experience and intuition had more influence on the conveyance decision than the standardised assessment tool, although high-risk patients who initially refused conveyance were more likely to agree if a checklist tool was used. ${ }^{49}$

\section{Theme 4: input of significant others}

Consulting a physician, either by the EMS staff or by the patient, influenced conveyance rates. When a patient initially refused transport to the hospital, contact with a physician could change the decision in favour of conveyance to the ED. ${ }^{49} 5054$ Telephone discussions between the paramedic, patient and an EMS physician led in one study to a major reduction in ED conveyance rate and in the median response time (from notification to ambulance back in service). ${ }^{71}$ Another study similarly found that when EMS staff were unable to consult a physician, the patient was more likely to be conveyed to the $\mathrm{ED}^{63}$ Research investigating partnerships between general practitioners (GPs) and EMS staff showed that face-to-face contact between GP and patient led to lower conveyance rates than when the GP support was only by telephone. ${ }^{6776}$

Consulting a colleague or other healthcare provider (members of teams specialising in falls) was also mentioned as a factor that could prevent unnecessary conveyance to the $\mathrm{ED} .{ }^{63}$

Two studies reported that confident EMS staff were steered by the views of a patient (known to suffer from epilepsy) and believed that the patient understood their situation sufficiently well to be able to make the decision for themselves. ${ }^{5266}$

When responding to patients in end-of-life situations, EMS staff would prefer to meet the wishes of the patient if a patient had the capacity for decision-making or if the situation was correctly documented. ${ }^{66}$

Finally, there is the influence of the dispatcher. EMS crews reported that the information provided by the dispatcher could frame their expectations and influence the decision-making. ${ }^{63} 73$

\section{Theme 5: judgement}

Judgement of contextual factors can be used to gather information to support decision-making. A decision to convey to the ED could be influenced by others. Strong reactions from family members, carers or bystanders were mentioned as a reason to prevent or stabilise a crisis and choose the safest option. ${ }^{52} 5864$ In addition, any dissatisfaction by the patient or their family due to a lengthy response time was mentioned as a factor leading to conveyance to alleviate the situation. ${ }^{62}$

Sometimes, paramedics can seek confirmation from their colleague, and one could be influenced by the other. There were also situations where the colleague had an alternative approach to theirs, including conveying patients against their perceived best interests. ${ }^{66}$

When non-conveyance is being considered as an option, the EMS staff take into account whether someone should and could be involved in taking further care of the patient. The presence of adequate care/carers was reported as having an influence on this decision. ${ }^{61} 69$

\section{Conceptual framework}

The process of data extraction and coding led to a small revision of the framework. The theme 'Decisions' was redefined as 'Input of significant others', in order to give a more accurate description of the factors found from the studies. 'Use of decision support tools' was added as a new theme. No factors were found related to the theme 'evaluation' and is therefore removed from the conceptual framework. Factors linked to 'outcomes' were displayed as objective and subjective outcomes. The revised conceptual framework is displayed in figure 3.

\section{DISCUSSION}

\section{Summary of evidence}

The main aim of this MSR was to provide insight and a deeper understanding of factors that influence the decision regarding conveyance of elderly patients to an ED after an emergency ambulance attendance. Further, we looked at both objective and subjective outcomes related to the conveyance decision such as the occurrence of 


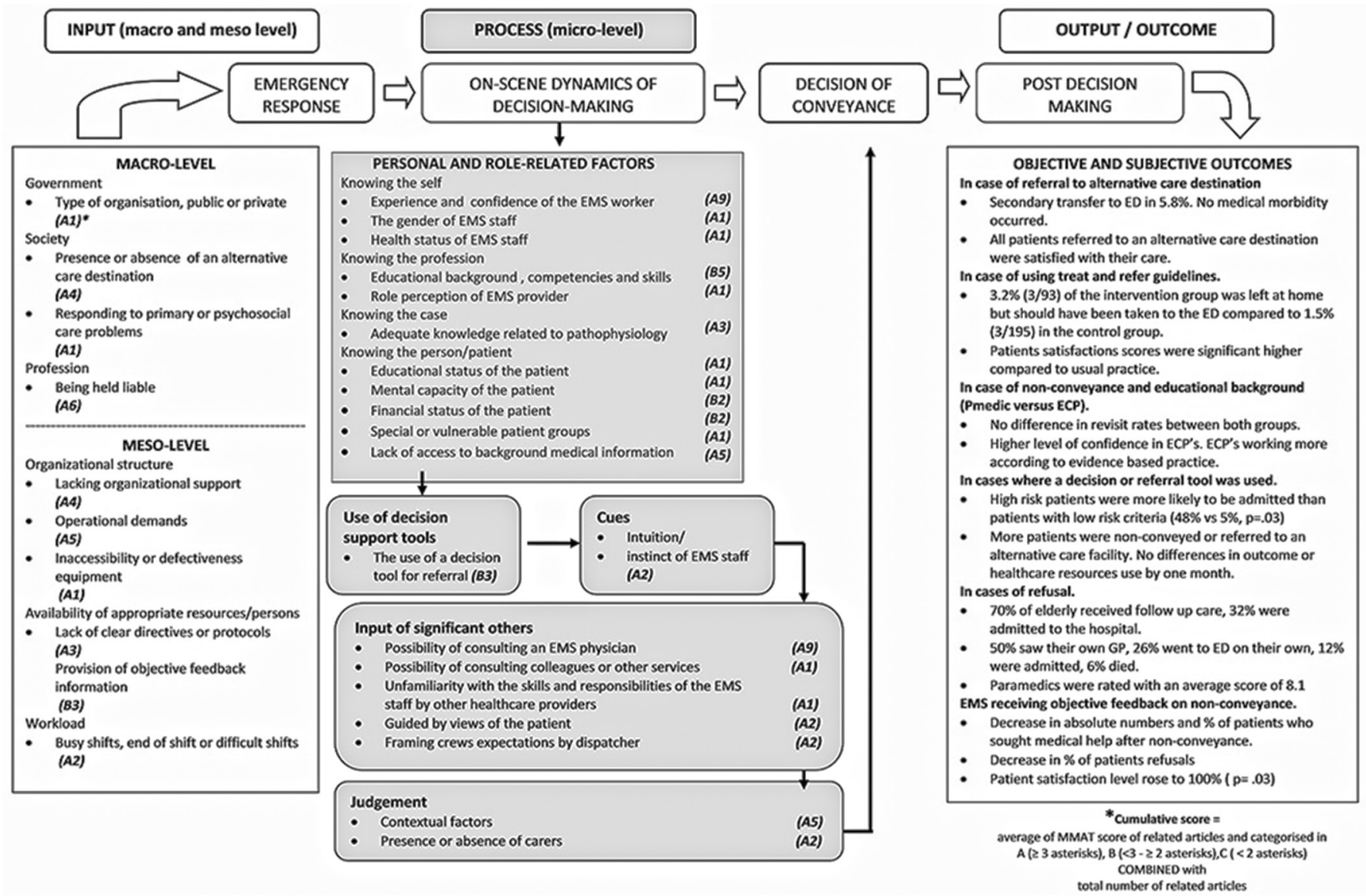

Figure 3 Conceptual framework of factors affecting the decision of ambulance service personnel regarding conveying adult patients to an emergency department. ED, emergency department; ECP, emergency care practitioner; EMS, emergency medical service; GP, general practitioner; MMAT, mixed-methods appraisal tool; Pmedic, paramedic.

undesirable outcomes and patient-reported outcomes. Findings are presented in an overarching framework that primarily reflects the relatively large influence of factors unrelated to a patient's condition on the conveyance decision.

Decisions over whether to convey someone to an ED after an emergency ambulance attendance often concern elderly people. An incorrect decision over an elderly person can lead to an increased risk of adverse or health-threatening effects as a result of chronic or multiple diseases, frailty, disability, polypharmacy and social isolation. ${ }^{20} 21242577$ Consequently, we decided to focus on the elderly in this study. We found 8 of the 29 studies included in our review primarily focused on elderly patients. Most of the studies focused on elderly were related to conveyance decisions after a fall. The presence or absence of informal carers was mentioned as factor influencing the conveyance decision. In the absence of informal carers, elderly patients are likely to be taken to an ED even if there is no underlying life-threatening condition. These avoidable referrals to the ED can be hazardous, especially for vulnerable elderly people, and puts an additional strain on those treating a large number of acute admissions to the ED, and its resources, and also leads to higher healthcare costs. ${ }^{3878}$
When broadening our scope and including all age groups, our first relevant finding is that the majority of factors that influence the conveyance decision are not determined by the direct contact between patient and EMS staff. Mainly on the macro and meso levels, and in personal and role-related factors, a variety of non-medical factors are influential. Our review of the literature shows that EMS staff are more likely to decide to convey a patient to the ED if they perceive a lack of organisational support, lack access to, or have defective, equipment, have counteracting performance indicators or sense that they are being held responsible for a patient's health. These findings indicate the relevance of patient-unrelated factors in conveyance decisions that might have a significant impact on patient safety, resource use and, ultimately, healthcare costs. Being held liable while, at the same time, experiencing insufficient organisational support and a 'shame and blame' culture can obstruct organisational learning and patient safety, whereas boosting the competences and working conditions of healthcare staff and leadership are known to increase the quality of healthcare ${ }^{79-81}$ When managers are aware that macro and meso factors can have a major impact on conveyance decisions, and act accordingly, EMS staff can make more effective and efficient decisions. 
Several factors, from both the EMS staff and patient perspectives, have been identified as affecting the conveyance decision-making process. Work experience, and its impact on the confidence of EMS professionals, was often cited as a factor that influenced the conveyance decision. Research on registered nurses in hospitals has similarly demonstrated a positive link between work experience and competence, and showed this had an influence on patient outcomes. ${ }^{82}$ Higher education levels, permanent employment and participation in educational programmes also boosted employees' feelings of competence. ${ }^{82}$ As such, investing in increasing the knowledge and skills needed to assess the elderly, and in the expanding options for non-emergency responses, would seem to pay off. Introducing EMS staff with additional specialised knowledge and competences regarding elderly care could improve on-scene care and avoid unnecessary ED admissions. Here, our MSR shows that EMS specialists were more likely to treat patients at the scene than paramedics, although there was little evidence in terms of different outcomes during the follow-up period. ${ }^{53} 73$ Further exploring the effect of using EMS specialists in assessing, treating and referring elderly patients should be considered and linked to objective and subjective outcomes.

EMS staff can find it helpful if they can contact a physician in questionable and doubtful situations since this may provide EMS staff with the necessary medical information to make a correct referral decision. On the micro level, we saw that enabling EMS staff to consult a physician could increase the likelihood of conveying, possibly overlooked, high-risk patients and a decrease in unnecessary referrals of non-emergency cases to the ED. ${ }^{49}$ There are also multiple studies that describe how contacting a physician (EMS physician or GP) has a positive influence in cases where a patient initially refuses transfer to the ED. ${ }^{49} 5054$ Facilities such as telecare and telehealth can support this consultation process and could be further investigated in order to improve the decision-making process.

A recent systematic review provided us with considerable data on the outcomes of a decision not to convey a patient to the ED. ${ }^{16}$ The researchers concluded that, after non-conveyance, $6.1 \%$ of the patients again contacted EMS within 24 hours, and up to $19 \%$ visited an ED within 48 hours of the initial interaction. In our MSR, we found evidence that being able to refer to alternative care facilities, using EMS specialists (ECPs), using referral tools, providing objective feedback to EMS staff and enabling EMS staff to contact a physician were all feasible and safe options to increase the likelihood that patients received the right care in the appropriate place.

However, we also found several factors leading to referrals to the ED when alternative care destinations or non-referral could be a better option. Despite there being a lack of research on the proportion of patients being conveyed while not strictly requiring hospital care, previous research shows that such a decision comes with risks and disadvantages, such as increased pressure on the ED, longer and often overnight stays in the ED and hospital, which all add to costs. ${ }^{21} 23-2538$ To improve the future quality of EMS responses, more data are needed on avoidable conveyance decisions, in terms of the actual numbers, and subsequent research on how to reduce this.

\section{LIMITATIONS}

A possible weakness is that the factors identified cannot be assumed to relate to elderly people because, in many studies, the elderly were just part of a broader study population, and the results were not specified by age group. In addition, the low methodological quality in some of the studies and the considerable age of some of them are also limitations of the study.

\section{CONCLUSIONS}

Making a decision to convey an elderly person to the hospital after an emergency ambulance response is not only determined by the assessment of medical conditions, but additional factors also influences this decision. These factors should be taken into account when new guidelines are being developed, or when new research is conducted into conveyance decisions, to ensure that greater insight will be developed on how multiple factors and their interplay influence the conveyance decision. Given the rapidly increasing number of vulnerable elderly individuals, it is, from both social and medical perspectives, highly relevant that EMS responses avoid unnecessary hospitalisation, and that evidence is provided to support future safe conveyance guidelines.

\section{IMPLICATIONS FOR FUTURE RESEARCH}

The low methodological quality in some of the studies, the considerable age of some of them and the broader population covered in many of them mean that further research focused on exploring the factors found in this review within EMS practice and the population of elderly people is warranted. In addition, study could be carried out to quantify the occurrence of preventable admissions to EDs based on the factors identified in this review.

\footnotetext{
Author affiliations

${ }^{1}$ Department of Health Sciences - Nursing Research, UMC Groningen, Groningen, The Netherlands

${ }^{2} \mathrm{NHL}$ Stenden, University of Applied Sciences, Leeuwarden, The Netherlands ${ }^{3}$ Ambulance Department, University Medical Center Groningen, Roden, The Netherlands

${ }^{4}$ Research Department of Emergency and Critical Care, HAN University of Applied Sciences, Faculty of Health and Social Studies, Nijmegen, The Netherlands

${ }^{5}$ Eastern Regional Emergency Healthcare Network, Radboud University Medical Centre, Nijmegen, The Netherlands

${ }^{6}$ IQ Healthcare, Radboud Institute for Health Sciences, Radboud University Medical Center, Nijmegen, The Netherlands

${ }^{7}$ Operations Department, Faculty of Economics and Business, Groningen, The Netherlands
} 
Acknowledgements We would like to thank Giles Stacey for his detailed comments on the manuscript.

Contributors All the authors contributed to the study design. Data were collected and selected by JO, DS and MB. Critical appraisal of all the selected articles was divided among PR, DS and MB. JO independently reviewed all the articles and results were compared. Agreement on a definitive appraisal was obtained by discussion. The manuscript was drafted by J0 in close collaboration with MB and SB. All the authors approved the final manuscript before submitting.

Funding The authors have not declared a specific grant for this research from any funding agency in the public, commercial or not-for-profit sectors.

Competing interests None declared.

Patient consent Not required.

Provenance and peer review Not commissioned; externally peer reviewed.

Data sharing statement Additional documentation on the data-collection process and appraisal is available from the corresponding author on reasonable request.

Open access This is an open access article distributed in accordance with the Creative Commons Attribution Non Commercial (CC BY-NC 4.0) license, which permits others to distribute, remix, adapt, build upon this work non-commercially, and license their derivative works on different terms, provided the original work is properly cited, appropriate credit is given, any changes made indicated, and the use is non-commercial. See: http://creativecommons.org/licenses/by-nc/4.0/.

\section{REFERENCES}

1. Lowthian JA, Cameron PA, Stoelwinder JU, et al. Increasing utilisation of emergency ambulances. Aust Health Rev 2011;35:63-9.

2. Lowthian JA, Jolley DJ, Curtis AJ, et al. The challenges of population ageing: accelerating demand for emergency ambulance services by older patients, 1995-2015. Med J Aust 2011;194:574-8.

3. Munjal KG, Silverman RA, Freese J, et al. Utilization of emergency medical services in a large urban area: description of call types and temporal trends. Prehosp Emerg Care 2011;15:371-80.

4. Peacock PJ, Peacock JL, Victor CR, et al. Changes in the emergency workload of the London ambulance service between 1989 and 1999. Emerg Med J 2005;22:56-9.

5. Clark MJ, Purdie J, FitzGerald GJ, et al. Predictors of demand for emergency prehospital care: an Australian study. Prehosp Disaster Med 1999;14:60-6.

6. Svenson JE. Patterns of use of emergency medical transport: a population-based study. Am J Emerg Med 2000;18:130-4.

7. Burt CW, McCaig LF, Valverde RH. Analysis of ambulance transports and diversions among US emergency departments. Ann Emerg Med 2006;47:317-26.

8. Metcalfe J. Enhancing the care of older people in the community. Nurs Stand 2006;21:35-9.

9. Logan PA, Coupland CA, Gladman JR, et al. Community falls prevention for people who call an emergency ambulance after a fall: randomised controlled trial. BMJ 2010;340:c2102.

10. Gray JT, Walker A. Avoiding admissions from the ambulance service: a review of elderly patients with falls and patients with breathing difficulties seen by emergency care practitioners in South Yorkshire. Emerg Med J 2008;25:168-71.

11. Snooks HA, Halter M, Close JC, et al. Emergency care of older people who fall: a missed opportunity. Qual Saf Health Care 2006;15:390-2.

12. Ebben $\mathrm{RH}$, Vloet LC, van Grunsven PM, et al. Factors influencing ambulance nurses' adherence to a national protocol ambulance care: an implementation study in the Netherlands. Eur J Emerg Med 2015;22:199-205.

13. Samaras N, Chevalley T, Samaras D, et al. Older patients in the emergency department: a review. Ann Emerg Med 2010;56:261-9.

14. Hjälte L, Suserud BO, Herlitz J, et al. Why are people without medical needs transported by ambulance? A study of indications for prehospital care. Eur J Emerg Med 2007;14:151-6.

15. Victor CR, Peacock JL, Chazot C, et al. Who calls 999 and why? A survey of the emergency workload of the London Ambulance Service. J Accid Emerg Med 1999;16:174-8.

16. Ebben RHA, Vloet LCM, Speijers RF, et al. A patient-safety and professional perspective on non-conveyance in ambulance care: a systematic review. Scand J Trauma Resusc Emerg Med 2017;25:71.

17. Schmidt MJ, Handel D, Lindsell CJ, et al. Evaluating an emergency medical services-initiated nontransport system. Prehosp Emerg Care 2006;10:390-3.
18. Tiedemann A, Mikolaizak AS, Sherrington C, et al. Older fallers attended to by an ambulance but not transported to hospital: a vulnerable population at high risk of future falls. Aust N Z J Public Health 2013;37:179-85.

19. Zachariah BS, Bryan D, Pepe PE, et al. Follow-up and outcome of patients who decline or are denied transport by EMS. Prehosp Disaster Med 1992;7:359-64.

20. Creditor MC. Hazards of hospitalization of the elderly. Ann Intern Med 1993;118:219-23.

21. Covinsky KE, Palmer RM, Fortinsky RH, et al. Loss of independence in activities of daily living in older adults hospitalized with medical illnesses: increased vulnerability with age. J Am Geriatr Soc 2003;51:451-8.

22. Schull MJ, Vermeulen M, Slaughter G, et al. Emergency department crowding and thrombolysis delays in acute myocardial infarction. Ann Emerg Med 2004;44:577-85.

23. Wilber ST, Gerson LW, Terrell KM, et al. Geriatric emergency medicine and the 2006 institute of medicine reports from the committee on the future of emergency care in the U.S. health system. Acad Emerg Med 2006;13:1345-51.

24. Richardson DB. Increase in patient mortality at 10 days associated with emergency department overcrowding. Med J Aust 2006;184:213-6.

25. McMullan JT, Veser FH. Emergency department volume and acuity as factors in patients leaving without treatment. South Med J 2004;97:729-33.

26. Snooks HA, Dale J, Hartley-Sharpe C, et al. On-scene alternatives for emergency ambulance crews attending patients who do not need to travel to the accident and emergency department: a review of the literature. Emerg Med J 2004;21:212-5.

27. Patterson PD, Moore CG, Brice JH, et al. Use of ED diagnosis to determine medical necessity of EMS transports. Prehosp Emerg Care 2006;10:488-93.

28. Arts DL, Voncken AG, Medlock S, et al. Reasons for intentional guideline non-adherence: a systematic review. Int J Med Inform 2016;89:55-62.

29. van de Glind I, Berben S, Zeegers F, et al. A national research agenda for pre-hospital emergency medical services in the Netherlands: a Delphi-study. Scand J Trauma Resusc Emerg Med 2016;24:2.

30. Ebben RH, Vloet LC, Schalk DM, et al. An exploration of factors influencing ambulance and emergency nurses' protocol adherence in the Netherlands. J Emerg Nurs 2014;40:124-30.

31. Mikolaizak AS, Simpson PM, Tiedemann A, et al. Systematic review of non-transportation rates and outcomes for older people who have fallen after ambulance service call-out. Australas J Ageing 2013;32:147-57.

32. Brown LH, Hubble MW, Cone DC, et al. Paramedic determinations of medical necessity: a meta-analysis. Prehosp Emerg Care 2009;13:516-27.

33. Miles J, O'Keeffe C, Jacques R, et al. 17 Exploring ambulance conveyances to the emergency department: a descriptive analysis of non-urgent transports. Emergency Medicine Journal 2017;34:A872-A873.

34. Snooks H, Cheung WY, Close J, et al. Support and Assessment for Fall Emergency Referrals (SAFER 1) trial protocol. Computerised on-scene decision support for emergency ambulance staff to assess and plan care for older people who have fallen: evaluation of costs and benefits using a pragmatic cluster randomised trial. BMC Emerg Med 2010;10:2.

35. Snooks H, Anthony R, Chatters R, et al. Support and assessment for fall emergency referrals (SAFER 2) research protocol: cluster randomised trial of the clinical and cost effectiveness of new protocols for emergency ambulance paramedics to assess and refer to appropriate community-based care. BMJ Open 2012;2:e002169.

36. Selden BS, Schnitzer PG, Nolan FX, et al. The "No-Patient" Run: 2,698 Patients Evaluated but Not Transported by Paramedics. Prehosp Disaster Med 1991;6:135-42.

37. Snooks HA. Gaps between policy, protocols and practice: a qualitative study of the views and practice of emergency ambulance staff concerning the care of patients with non-urgent needs. Quality and Safety in Health Care 2005;14:251-7.

38. Dwyer R, Gabbe B, Stoelwinder JU, et al. A systematic review of outcomes following emergency transfer to hospital for residents of aged care facilities. Age Ageing 2014;43:759-66.

39. Pluye P, Hong QN, Vedel I. Toolkit for mixed studies reviews (V3). Montreal, Canada: Department of Family Medicine, McGill University, and Quebec-SPOR SUPPORT Unit, 2016.

40. Pluye P, Hong QN. Combining the power of stories and the power of numbers: mixed methods research and mixed studies reviews. Annu Rev Public Health 2014;35:29-45. 
41. The laboratory of research on software engineering (LaPES). computing department of the federal university of são carlos. 2017. http://lapes.dc.ufscar.br/resources-and-downloads/tools

42. Pace R, Pluye P, Bartlett G, et al. Testing the reliability and efficiency of the pilot Mixed Methods Appraisal Tool (MMAT) for systematic mixed studies review. Int J Nurs Stud 2012;49:47-53.

43. Carroll C, Booth A, Leaviss J, et al. "Best fit" framework synthesis: refining the method. BMC Med Res Methodol 2013;13:37.

44. Gillespie M, Peterson BL. Helping novice nurses make effective clinical decisions: the situated clinical decision-making framework. Nurs Educ Perspect 2009;30:164-70.

45. Gillespie M. Using the situated clinical decision-making framework to guide analysis of nurses' clinical decision-making. Nurse Educ Pract 2010;10:333-40.

46. Steiner I. Group process and productivity. Academic Press 1972.

47. Hackman JR. The design of work teams. In: Lorsch J, ed. Handbook of organizational behavior. Englewood Cliffs, NJ: Prentice-Hall, 1987:315-42.

48. Persse DE, Key CB, Baldwin JB. The effect of a quality improvement feedback loop on paramedic-initiated nontransport of elderly patients. Prehosp Emerg Care 2002;6:31-5.

49. Alicandro J, Hollander JE, Henry MC, et al. Impact of interventions for patients refusing emergency medical services transport. Acad Emerg Med 1995;2:480-5.

50. Vilke GM, Sardar W, Fisher R, et al. Follow-up of elderly patients who refuse transport after accessing 9-1-1. Prehosp Emerg Care 2002;6:391-5.

51. Aftyka A, Rudnicka-Drożak E, Rybojad B. A comparison of ambulance responses to incidents of Medical Emergency Teams led by nurses and paramedics-A retrospective single-center study. Int $J$ Nurs Stud 2014;51:555-61.

52. Burrell L, Noble A, Ridsdale L. Decision-making by ambulance clinicians in London when managing patients with epilepsy: a qualitative study. Emerg Med J 2013;30:236-40.

53. Cooper S, Barrett B, Black S, et al. The emerging role of the emergency care practitioner. Emerg Med J 2004;21:614-8.

54. Stuhlmiller DF, Cudnik MT, Sundheim SM, et al. Adequacy of online medical command communication and emergency medical services documentation of informed refusals. Acad Emerg Med 2005;12:970-7.

55. Snooks HA, Carter B, Dale J, et al. Support and assessment for fall emergency referrals (SAFER 1): cluster randomised trial of computerised clinical decision support for paramedics. PLoS One 2014;9:e106436

56. Waldron R, Finalle C, Tsang J, et al. Effect of gender on prehospital refusal of medical aid. J Emerg Med 2012;43:283-90.

57. Waldrop DP, Clemency B, Lindstrom HA, et al. "We are strangers walking into their life-changing event": how prehospital providers manage emergency calls at the end of life. J Pain Symptom Manage 2015:50:328-34.

58. Waldrop DP, Clemency B, Maguin E, et al. Preparation for frontline end-of-life care: exploring the perspectives of paramedics and emergency medical technicians. J Palliat Med 2014;17:338-41.

59. Zorab O, Robinson M, Endacott R. Are prehospital treatment or conveyance decisions affected by an ambulance crew's ability to access a patient's health information? BMC Emerg Med 2015;15:26.

60. Burstein JL, Hollander JE, Delagi R, et al. Refusal of out-of-hospital medical care: effect of medical-control physician assertiveness on transport rate. Acad Emerg Med 1998;5:4-8.

61. Snooks HA, Kearsley N, Dale J, et al. Gaps between policy, protocols and practice: a qualitative study of the views and practice of emergency ambulance staff concerning the care of patients with non-urgent needs. Qual Saf Health Care 2005;14:251-7.

62. Ebrahimian A, Seyedin H, Jamshidi-Orak R, et al. Exploring factors affecting emergency medical services staffs' decision about transporting medical patients to medical facilities. Emerg Med Int 2014;2014:1-8

63. O'Hara R, Johnson M, Siriwardena AN, et al. A qualitative study of systemic influences on paramedic decision making: care transitions and patient safety. J Health Serv Res Policy 2015;20(1 Suppl):45-53.

64. Porter A, Snooks H, Youren A, et al. Should I stay or should I go?' Deciding whether to go to hospital after a 999 call. J Health Serv Res Policy 2007;12:32-8.

65. Langabeer JR, Gonzalez M, Alqusairi D, et al. Telehealth-Enabled emergency medical services program reduces ambulance transport to urban emergency departments. West J Emerg Med 2016;17:713-20.

66. Murphy-Jones G, Timmons S. Paramedics' experiences of end-oflife care decision making with regard to nursing home residents: an exploration of influential issues and factors. Emerg Med $J$ 2016;33:722-6.

67. Williams JG, Bachman MW, Lyons MD, et al. Improving decisions about transport to the emergency department for assisted living residents who fall. Ann Intern Med 2018;168:179.

68. Schaefer RA, Rea TD, Plorde M, et al. An emergency medical services program of alternate destination of patient care. Prehosp Emerg Care 2002;6:309-14.

69. Halter M, Vernon S, Snooks H, et al. Complexity of the decisionmaking process of ambulance staff for assessment and referral of older people who have fallen: a qualitative study. Emerg Med $J$ 2011;28:44-50.

70. Déziel J. Effects of emergency medical services agency ownership status on patient transport. Prehosp Emerg Care 2017;21:729-33.

71. Larsson G, Holmén A, Ziegert K. Early prehospital assessment of non-urgent patients and outcomes at the appropriate level of care: $A$ prospective exploratory study. Int Emerg Nurs 2017;32:45-9.

72. Noble AJ, Snape D, Goodacre S, et al. Qualitative study of paramedics' experiences of managing seizures: a national perspective from England. BMJ Open 2016;6:e014022.

73. Simpson P, Thomas R, Bendall J, et al. 'Popping nana back into bed' - a qualitative exploration of paramedic decision making when caring for older people who have fallen. BMC Health Serv Res 2017;17.

74. Snooks HA, Anthony R, Chatters R, et al. Paramedic Assessment of Older Adults After Falls, Including Community Care Referral Pathway: Cluster Randomized Trial. Ann Emerg Med 2017;70:495-505.

75. Snooks H, Kearsley N, Dale J, et al. Towards primary care for non-serious 999 callers: results of a controlled study of "Treat and Refer" protocols for ambulance crews. Qual Saf Health Care 2004;13:435-43.

76. Villarreal M, Leach J, Ngianga-Bakwin K, et al. Can a partnership between general practitioners and ambulance services reduce conveyance to emergency care? Emerg Med J 2017;34:459-65.

77. Hwang $\mathrm{U}$, Shah MN, Han JH, et al. Transforming emergency care for older adults. Health Aff 2013;32:2116-21.

78. van der Linden N, van der Linden MC, Richards JR, et al. Effects of emergency department crowding on the delivery of timely care in an inner-city hospital in the Netherlands. Eur J Emerg Med 2016;23:337-43.

79. Almeida SL. Nursing perspectives on the emergency department. Emerg Med Clin North Am 2004;22117-.

80. Robinson KS, Jagim MM, Ray CE. Nursing workforce issues and trends affecting emergency departments. Nurs Manage 2005;36:46-53.

81. Raup GH. The impact of ED nurse manager leadership style on staff nurse turnover and patient satisfaction in academic health center hospitals. J Emerg Nurs 2008;34:403-9.

82. Flinkman $\mathrm{M}$, Leino-Kilpi $\mathrm{H}$, Numminen $\mathrm{O}$, et al. Nurse competence scale: a systematic and psychometric review. J Adv Nurs 2017;73:1035-50. 